\title{
The mystery of photometric twins DES17X1boj and DES16E2bjy
}
M. Pursiainen ${ }^{\odot},{ }^{1 \star}$ C. P. Gutiérrez, ${ }^{1}$ P. Wiseman ${ }^{\oplus},{ }_{1}^{1}$ M. Childress ${ }^{\oplus},{ }^{1}$ M. Smith ${ }^{\oplus}, 1$
C. Frohmaier ${ }^{\oplus},{ }^{2}$ C. Angus ${ }^{\oplus},{ }^{1}$ N. Castro Segura, ${ }^{1}$ L. Kelsey ${ }^{\odot},{ }^{1}$ M. Sullivan, ${ }^{1}$
L. Galbany ${ }^{\odot},{ }^{3}$ P. Nugent, ${ }^{4}$ B. A. Bassett, ${ }^{5,6}$ D. Brout, ${ }^{7}$ D. Carollo,${ }^{8}$ C. B. D’Andrea, ${ }^{7}$
T. M. Davis, ${ }^{9}$ R. J. Foley, ${ }^{10}$ M. Grayling, ${ }^{1}$ S. R. Hinton, ${ }^{9}$ C. Inserra, ${ }^{11}$ R. Kessler, ${ }^{12,13}$
G. F. Lewis, ${ }^{14}$ C. Lidman, ${ }^{15}$ E. Macaulay, ${ }^{2}$ M. March, ${ }^{7}$ A. Möller, ${ }^{16}$ T. Müller, ${ }^{1}$
D. Scolnic, ${ }^{17}$ N. E. Sommer, ${ }^{15}$ E. Swann, ${ }^{2}$ B. P. Thomas, ${ }^{18}$ B. E. Tucker, ${ }^{15}$
M. Vincenzi, ${ }^{2}$ T. M. C. Abbott, ${ }^{19}$ S. Allam, ${ }^{20}$ J. Annis, ${ }^{20}$ S. Avila, ${ }^{21}$ E. Bertin, ${ }^{22,23}$
D. Brooks, ${ }^{24}$ E. Buckley-Geer, ${ }^{20}$ D. L. Burke, ${ }^{25,26}$ A. Carnero Rosell, ${ }^{27,28}$ M. Carrasco
Kind, ${ }^{29,30}$ L. N. da Costa, ${ }^{28,31}$ J. De Vicente, ${ }^{27}$ S. Desai, ${ }^{32}$ H. T. Diehl, ${ }^{20}$ P. Doel, ${ }^{24}$
T. F. Eifler, ${ }^{33,34}$ S. Everett, ${ }^{10}$ B. Flaugher,${ }^{20}$ J. Frieman, ${ }^{20,13}$ J. García-Bellido, ${ }^{21}$
E. Gaztanaga, ${ }^{35,36}$ D. W. Gerdes, ${ }^{37,38}$ D. Gruen, ${ }^{25,26,39}$ R. A. Gruendl, ${ }^{29,30}$
J. Gschwend ${ }^{28,31}$ G. Gutierrez ${ }^{\odot},{ }^{20}$ D. L. Hollowood, ${ }^{10}$ K. Honscheid, ${ }^{40,41}$
D. J. James, ${ }^{42}$ A. G. Kim, ${ }^{4}$ E. Krause, ${ }^{33}$ K. Kuehn, ${ }^{43,44}$ M. A. G. Maia, ${ }^{28,31}$
J. L. Marshall, ${ }^{45}$ F. Menanteau, ${ }^{29,30}$ R. Miquel, ${ }^{46,47}$ R. L. C. Ogando, ${ }^{28,31}$

A. Palmese,${ }^{20,13}$ F. Paz-Chinchón, ${ }^{29,30}$ A. A. Plazas, ${ }^{48}$ A. Roodman, ${ }^{25,26}$ E. Sanchez, ${ }^{27}$

V. Scarpine, ${ }^{20}$ M. Schubnell, ${ }^{38}$ S. Serrano, ${ }^{35,36}$ I. Sevilla-Noarbe, ${ }^{27}$ E. Suchyta, ${ }^{49}$

M. E. C. Swanson, ${ }^{30}$ G. Tarle, ${ }^{38}$ W. Wester ${ }^{20}$ and (DES Collaboration)

Affiliations are listed at the end of the paper

Accepted 2020 April 7. Received 2020 March 13; in original form 2019 November 26

\begin{abstract}
We present an analysis of DES17X1boj and DES16E2bjy, two peculiar transients discovered by the Dark Energy Survey (DES). They exhibit nearly identical double-peaked light curves that reach very different maximum luminosities $\left(M_{r}=-15.4\right.$ and -17.9 , respectively). The light-curve evolution of these events is highly atypical and has not been reported before. The transients are found in different host environments: DES17X1boj was found near the nucleus of a spiral galaxy, while DES16E2bjy is located in the outskirts of a passive red galaxy. Early photometric data are well fitted with a blackbody and the resulting moderate photospheric expansion velocities (1800 $\mathrm{km} \mathrm{s}^{-1}$ for DES17X1boj and $4800 \mathrm{~km} \mathrm{~s}^{-1}$ for DES16E2bjy) suggest an explosive or eruptive origin. Additionally, a feature identified as high-velocity Ca II absorption $\left(v \approx 9400 \mathrm{~km} \mathrm{~s}^{-1}\right)$ in the near-peak spectrum of DES17X1boj may imply that it is a supernova. While similar light-curve evolution suggests a similar physical origin for these two transients, we are not able to identify or characterize the progenitors.
\end{abstract}

Key words: supernovae: general.

\section{INTRODUCTION}

Dedicated wide-field supernova ( $\mathrm{SN}$ ) surveys have discovered large numbers of extragalactic transients over the last decade. A large

^E-mail: m.a.pursiainen@soton.ac.uk majority of them are traditional types of SNe, including Type Ia SNe (SNe Ia) produced in thermonuclear disruptions of white dwarfs and SNe II/Ibc originating in the aftermath of the core-collapse of massive stars ( $\gtrsim 8 \mathrm{M}_{\odot}$; see e.g. Filippenko 1997, Gal-Yam 2017 for reviews). However, due to the increase in area, cadence and depth, these surveys have also started to discover rarer types of events such as the rapidly evolving transients (e.g. Drout et al. 2014; Pursiainen 
et al. 2018), superluminous supernovae (SLSNe; e.g. Quimby et al. 2011; see Howell 2017 for a review), and Ca-rich transients (e.g. Perets et al. 2010), all of whose behaviour cannot be explained with the physical mechanisms used for typical SNe.

The Dark Energy Survey Supernova (DES-SN) Programme has proven to be an excellent laboratory for discovering unusual types of events with its deep ( $\sim 24$ mag per visit) multicolour griz photometry. It has, for example, already probed the diversity of a sample of SLSNe (Angus et al. 2019) with objects up to $z$ $\approx 2$ (DES16C2nm; Smith et al. 2018) and unveiled a sample of rapidly evolving transients (Pursiainen et al. 2018). In this paper, we present two additional peculiar transients discovered by DESSN, DES17X1boj, and DES16E2bjy, with highly atypical doublepeaked light curves nearly identical to each other.

Light curves exhibiting phases of re-brightening are not uncommon amongst extragalactic transients. SNe Ia have a characteristic secondary peak in the redder bands due to Fe III recombination (Kasen 2006), and a fraction of core-collapse SNe (CCSNe) and SLSNe also show similar behaviour. For example, several SNe IIb (e.g. SN1993J; Wheeler et al. 1993), SNe Ic iPTF14gqr (De et al. 2018), and several SLSNe (e.g. SN2006oz; Leloudas et al. 2012) all have short precursory bumps often attributed to shock cooling in extended material surrounding the SN. Additionally, several SNe IIP have shown shallow rising during their plateau phase (e.g. SN2009N; Takáts et al. 2014). However, none of these double-peaked SNe have similar light curves to the DES-SN transients, as they have differences in the duration and the strength of the two peaks.

Here we present an analysis of the photometric and spectroscopic data for the DES-SN photometric twins, DES17X1boj and DES16E2bjy. While they share nearly identical light-curve evolution, several other observable features are clearly different. The transients differ by nearly 3 mag in brightness with DES17X1boj peaking at $M_{r}=-15.4$ and DES16E2bjy reaching $M_{r}=-17.9$. They are also found in different host environments. DES17X1boj's position is consistent with the nucleus of a star-forming spiral galaxy, while DES16E2bjy appeared on the outskirts of a passive galaxy, with the only common factor being a presence of old stellar populations in both environments. While the high-velocity ( 9400 $\mathrm{km} \mathrm{s}^{-1}$ ) Ca II absorption feature in the spectrum of DES17X1boj and the photospheric expansion velocity of DES16E2bjy $(v \approx 4800$ $\mathrm{km} \mathrm{s}^{-1}$ ) strongly indicate an explosive or eruptive origin for these transients, the exact physical scenario remains unknown. Due to the several different observed features, it is also entirely possible that the transients do not share a similar origin, despite similar light-curve evolution.

In Section 2, we provide description of the observational data used in this paper, and in Sections 3 and 4, we present the analysis of the transients and their host galaxies. Finally, in Section 5, we discuss the physical scenarios that could explain the peculiar observed features of the transients and summarize our findings. Throughout this paper, we assume a flat Lambda cold dark matter $(\Lambda \mathrm{CDM})$ cosmology with $\Omega_{\mathrm{M}}=0.3$ and $H_{0}=70 \mathrm{~km} \mathrm{~s}^{-1} \mathrm{Mpc}^{-1}$.

\section{OBSERVATIONS}

DES17X1boj was first detected using the DIFFIMG pipeline (Kessler et al. 2015) at a signal-to-noise ratio $>5$ by DES-SN in Dark Energy Camera (DECam; Flaugher et al. 2015) griz images taken on 2017 October 9 at $\alpha=02^{\mathrm{h}} 19^{\mathrm{m}} 53^{\mathrm{s}} .80, \delta=-04^{\circ} 57^{\prime} 10^{\prime \prime} .4$ (J2000) with a previous non-detection on September 29. It reached a maximum brightness of $m_{r}=20.52\left(M_{r}=-15.35\right)$ on October 17 and was detected for the last time on December 13 of the same year. In the beginning of the light curve, DES17X1boj was identified as a potential candidate for a rapidly evolving transient in a live selection of targets during year five of DES-SN, where we visually inspected all new transients with relatively blue colours $(g-r<$ 0.2 ). Promptly after the discovery it was reclassified as another type of peculiar object due to the double-peaked light curve. The transient was observed spectroscopically for $2400 \mathrm{~s}$ on October 22 as part of the OzDES programme (Yuan et al. 2015; Childress et al. 2017; Lidman et al., in preparation) and the resulting data were reduced based on the pipeline described in Yuan et al. (2015). OzDES uses the 3.9-m Anglo-Australian Telescope (AAT) in Australia with the AAOmega spectrograph ande $2 \mathrm{dF}$ robotic fibre positioner. The resulting spectra has an average resolution of $R \sim 1400$, with $1 \AA$ pixel $^{-1}$ dispersion in the blue arm and $1.6 \AA \mathrm{pixel}^{-1}$ in the red arm (Yuan et al. 2015). As the underlying galaxy is 1.0-1.5 mag brighter than DES17X1boj at peak brightness in all bands, the spectrum is dominated by host galaxy features. The host of DES17X1boj was previously observed with the AAT under the Galaxy and Mass Assembly (GAMA; Driver et al. 2009; Baldry et al. 2018) programme for $7200 \mathrm{~s}$ in 2010 November.

DES16E2bjy was recovered with an archival search for transients that exhibited similar light-curve evolution to DES17X1boj that was performed after the end of DES-SN operations (see Section 2.1). The transient was first detected by DES-SN on 2016 September 21 at $\alpha=00^{\mathrm{h}} 33^{\mathrm{m}} 19^{\mathrm{s}} .34, \delta=-44^{\circ} 20^{\prime} 19^{\prime \prime} .6$ (J2000), with a previous nondetection on the 13th of September. It reached maximum brightness of $m_{r}=20.99\left(M_{r}=-17.94\right)$ on October 4 and was detected for the last time on December 17. DES16E2bjy was spectroscopically observed for $2400 \mathrm{~s}$ on October 5 by OzDES, and for 2800 s on 2016 October 7 with Robert Stobie Spectrograph (RSS; Nordsieck et al. 2001) on 10 meter-class South African Large Telescope (SALT) as it was a high-probability candidate for being an SN Ia. The SALT spectrum has a resolution of $R \sim 350$ and it was reduced using the PYSALT $^{1}$ pipeline (Crawford et al. 2010). All presented light curves have been corrected for the Milky Way extinction using colour excesses from Schlafly \& Finkbeiner (2011). Further details on the DES-SN difference imaging photometry can be found in Kessler et al. (2015) and on DES-SN spectroscopic follow-up programmes in D'Andrea et al. (2018). The photometric properties are presented in Section 3.1 and spectroscopic properties in Section 3.2.

The transient environments are presented in Fig. 1, with stamps created using the optimized DES-SN deep stacks (Wiseman et al. 2020). The location of DES17X1boj is consistent with the nucleus of a spiral galaxy (LEDA 1051269; Brouty et al. 2003) at $z=$ 0.0338 , while DES16E2bjy appears to be associated with a red elliptical galaxy at $z=0.1305$ with a separation of $\approx 5 \operatorname{arcsec}$ $(11.6 \mathrm{kpc})$. The host galaxy redshifts were obtained by the OzDES programme and all presented absolute magnitudes have been calculated based on them. The host galaxy properties are discussed in Section 4.

\subsection{Sample selection}

After DES17X1boj was discovered, an archival search was performed on the full $5 \mathrm{yr}$ of DES-SN data to search for transients with similar light-curve evolution. For this purpose, we generated interpolated light curves of all $~ 30000$ DES-SN transients using Gaussian processes (GP) with a similar approach to Angus et al.

\footnotetext{
${ }^{1}$ http://pysalt.salt.ac.za/.
} 

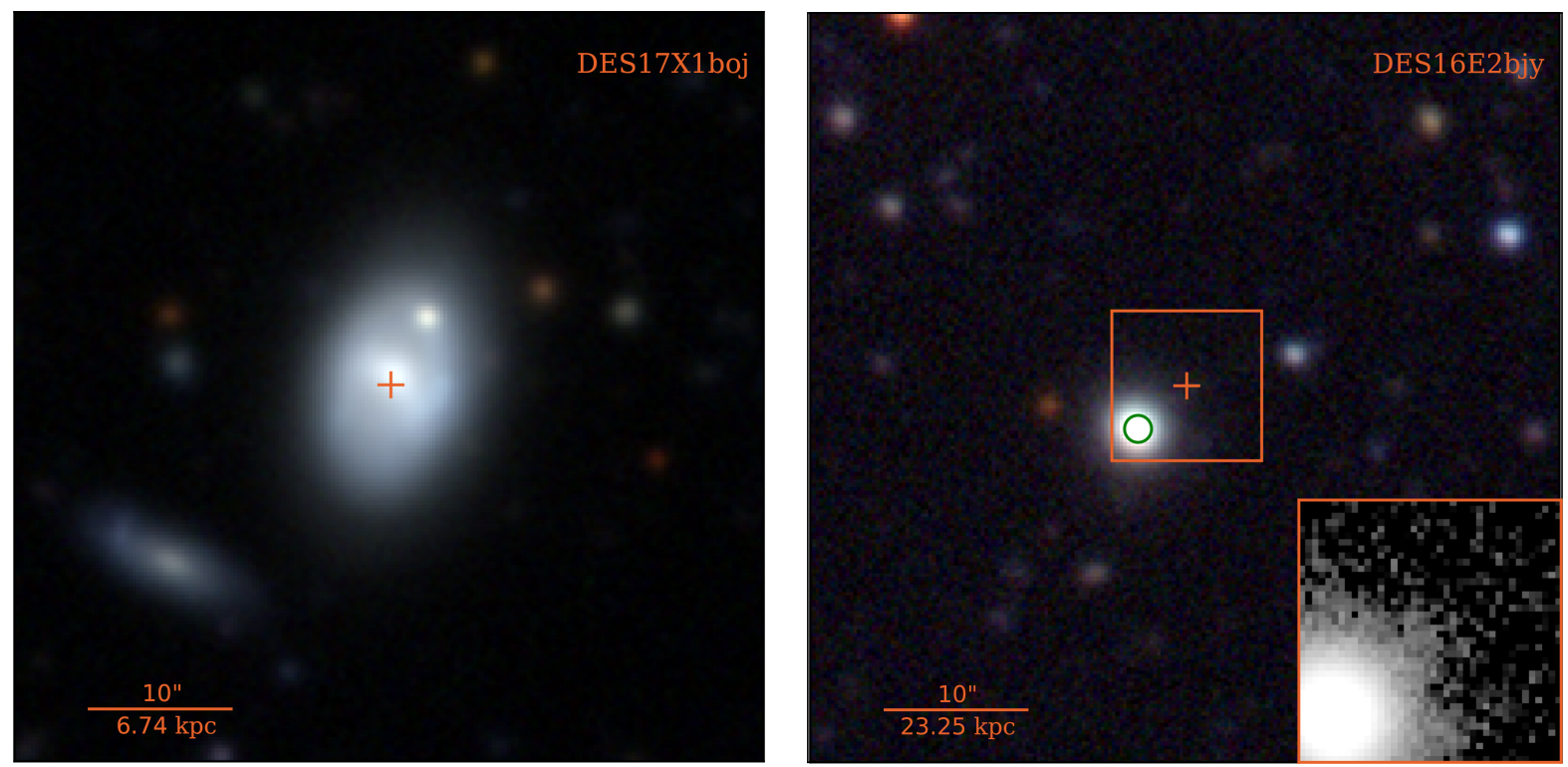

Figure 1. Host environments of DES17X1boj and DES16E2bjy. Transient locations are marked with red crosses and the assumed host of DES16E2bjy is marked with green circle. The host of DES17X1boj is at $z=0.0338$ and DES16E2bjy at $z=0.1305$. For DES16E2bjy, we also show a zoom-in of the $r$-band image around the transient in the bottom right-hand corner, revealing the presence of light presumably of the host galaxy at the location.

(2019) and Inserra et al. (2018). GP use the uncertainties of the flux measurements to determine how strongly sequential data points are correlated, and then interpolate the light curves based on a predefined function ('kernel') that defines how strongly the light curve may vary in time. In this paper, we use the squared exponential model for the kernel with time-scale of variation, which was determined using gradient-based optimization. The light curves were created with 0.5 -d cadence, so that every GP epoch had a flux value in all four bands. Every year and band was interpolated independently with the first epoch being at the time of the first observation of the year regardless of the band. The interpolation was performed with the PYTHON package GEORGE (Ambikasaran et al. 2016). Using the GP light curves, we performed a systematic search for DES-SN transients with similar light-curve evolution to DES17X1boj. In detail, the GP light curves were used to estimate the peak times and brightnesses of each named DES-SN transient so that we could easily find events fulfilling our search criteria.

We searched for all unclassified DES-SN transients for which a secondary peak of a 'boj-like' transient would have been seen (at least $24 \mathrm{mag}$ ). This requirement corresponds to brightness of the primary peak of at least 22 mag. However, we also constrained the the total observable volume by requiring the transient or its associated host galaxy to have a redshift $z \leq 0.2$. At this redshift the peak brightness of a transient observed at 22 mag would correspond to $M=-18$. With the given criteria, DES17X1boj $\left(M_{r}\right.$ $=-15.4$ ) could have been recovered only up to $z=0.07$. However, the search was designed to maximize our chance of discovering 'boj-like' transients while also allowing them to be brighter than DES17X1boj.

In total, 225 DES-SN transients passed the criteria. Light curves of these transients were visually inspected and only DES16E2bjy was found to have a similar double-peaked light-curve evolution as DES17X1boj. Most of the other inspected transients had light curves similar to the traditional SN types and the few remaining transients were found to be spurious detections.

\section{ANALYSIS}

\subsection{Photometric properties}

As shown in Fig. 2, DES17X1boj and DES16E2bjy share very similar light-curve evolution. Both rise to maximum brightness, as measured from photometry in the $g$ band, in 15-20 d and decline rapidly by $2-3 \mathrm{mag}$ in $\sim 20 \mathrm{~d}$ before plateauing and rising to the second peak-like feature. After the secondary peak both transients fade quickly below the detection limit. However, despite very similar light curves, the peak luminosities are very different: While DES17X1boj is $M_{r}=-15.4$ at observed maximum brightness, DES16E2bjy reaches $M_{r}=-17.9$. The presented light curves have not been $K$-corrected due to unknown spectral energy distributions (SEDs) during the secondary peak. However, we estimated the significance of $K$-correction at maximum brightness based on blackbody fits to the photometry (see Appendix A). The correction for DES17X1boj is less than $0.1 \mathrm{mag}$ and for DES16E2bjy $0.2-0.3$ mag so that each band becomes fainter.

The similarity of the light curves is further emphasized in Fig. 3, where a scaling of $2.5 \mathrm{mag}$ (factor of 10 in brightness) has been added to DES17X1boj to match the peak luminosities. The scaled light curves are nearly identical with the only significant difference between the two transients being the rise to maximum brightness, which appears to be longer for DES16E2bjy, especially in the bluer bands. The rise also appears to have two phases; a fast rise until $\approx 10 \mathrm{~d}$ before peak and a shallower, nearly linear rise to maximum brightness.

We have also plotted light curves of both normal and peculiar transients from the literature in Fig. 3 to demonstrate that the light-curve evolution of DES17X1boj and DES16E2bjy is highly atypical. The transients have been selected as they evolve in similar time-scales as the DES transients and show phases of rebrightening at similar epochs. SNe Ia (here illustrated with SN2011fe) always have a secondary peak but it is prominent only in the redder bands, while DES17X1boj and DES16E2bjy exhibit 

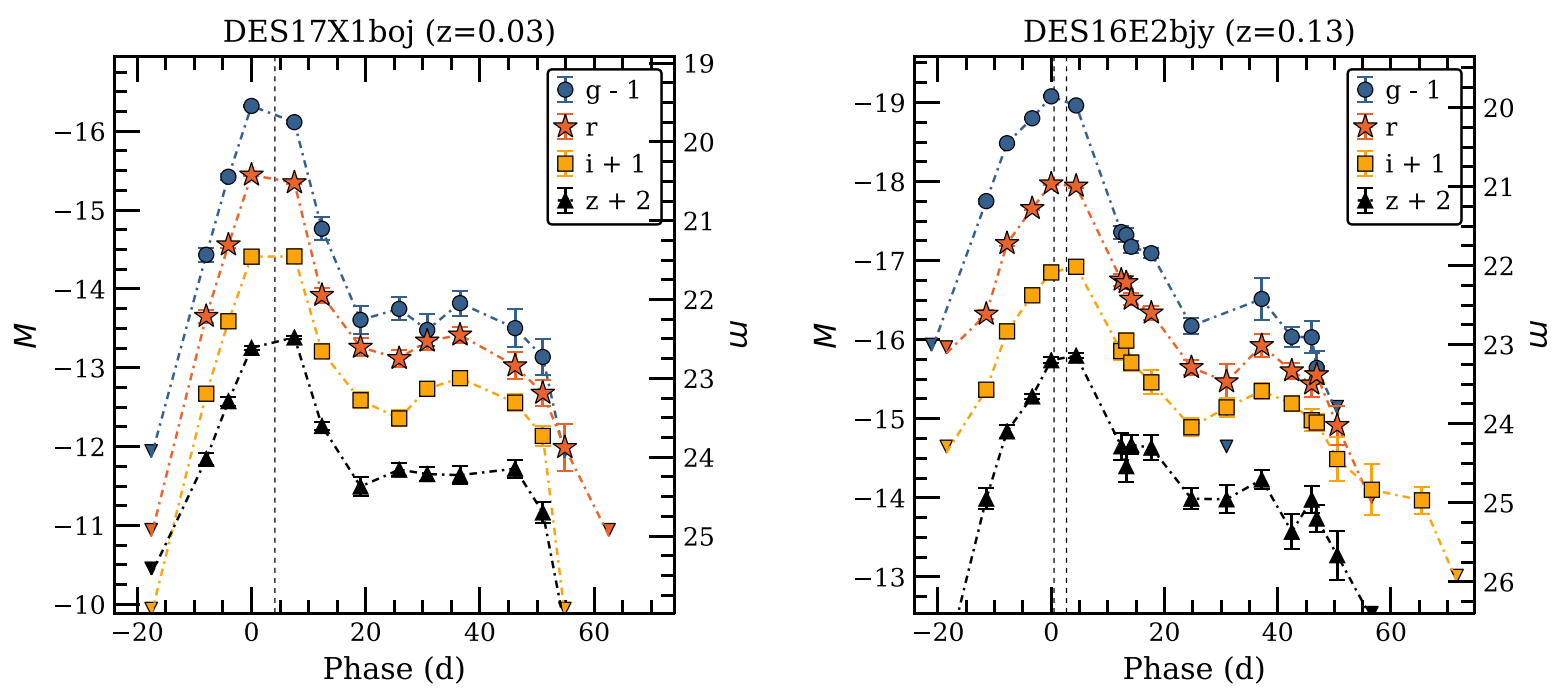

Figure 2. Rest-frame DES-SN $g, r, i, z$ light curves of DES17X1boj and DES16E2bjy, with respect to maximum brightness in the $g$ band. Photometric bands are offset for clarity. Downward-facing triangles represent upper limits. The phases of spectroscopic observations have been marked with dashed vertical lines.

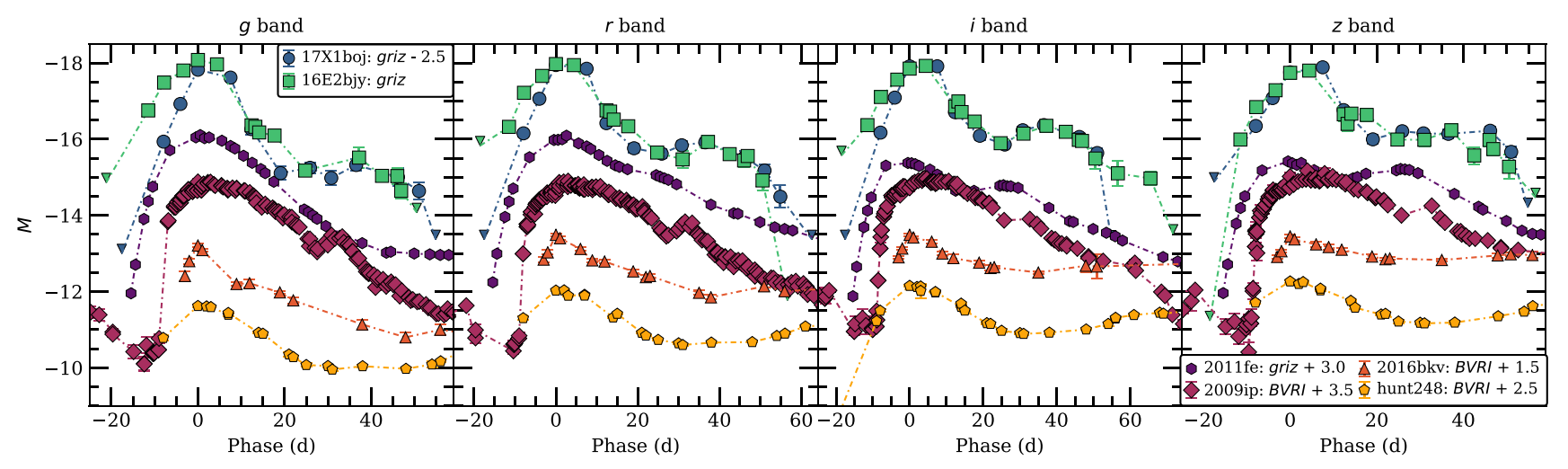

Figure 3. Light curves of DES16E2bjy and DES17X1boj, with respect to peak in the $g$ band, scaled by 2.5 mag to match in maximum brightness. SN Ia2011fe (Richmond \& Smith 2012; Munari et al. 2013; Tsvetkov et al. 2013), SN impostor SN2009ip (during the event in 2012; Fraser et al. 2013; Mauerhan et al. 2013; Pastorello et al. 2013; Brown et al. 2014), low-luminosity SN IIP2016bkv (Hosseinzadeh et al. 2018; Nakaoka et al. 2018), and triple-peaked transient event SNhunt248 (Kankare et al. 2015; Mauerhan et al. 2015), proposed to be a luminous red novae (LRNe; Mauerhan et al. 2018), have been plotted for comparison. The light curves of literature events were collected from The Open Supernova Catalogue (Guillochon et al. 2016), apart from SN2011fe for which the light curve was created based on BVRI light curves from the above sources that were $K$-corrected (Nugent, Kim \& Perlmutter 2002) using a spectrophotometric template from Hsiao et al. (2007). Note that offsets have been added to literature events for visual clarity.

a strong secondary peak in all four optical bands. Additionally, the shape of the secondary peaks seen in the $i$ and $z$ bands is very different from the DES-SN transients. Some SNe IIP, such as SN2016bkv, show shallow rising during their plateau phase, but on much longer time-scales and without significant changes in brightness. Even other peculiar transients with several phases of rebrightening, such as impostor SN2009ip (during the event in 2012) and LRN SNhunt248, do not show as extreme variation as seen in the light curves of DES17X1boj and DES16E2bjy.

We performed blackbody fits to each epoch of the four-band photometry for both of the transients and the fits are shown in Appendix A, Figs A1 and A2, for DES17X1boj and DES16E2bjy respectively. The photometric data are well described with a blackbody at least until $\sim 10 \mathrm{~d}$ after initial peak. At later times, the blackbody fits are less constrained due to fading targets. The blackbody temperature and radial evolution are shown in Fig. 4 with the observed $g-r$ colour evolution. DES16E2bjy is clearly bluer and therefore hotter in the beginning of the light curve, but after roughly $+10 \mathrm{~d}$, the transients share similar colour and temperature evolution. However, while DES162E2bjy starts with a high temperature of $T \approx 18000 \mathrm{~K}$ and quickly cools down, the temperature of DES17X1boj increases slightly until the peak brightness, reaching a temperature of $\approx 8000 \mathrm{~K}$. Afterwards, the temperature gradually decreases and there seems to be no evidence of significant colour evolution. On the other hand, the radii of the transients evolve similarly to each other. First, the radius increases nearly linearly until $\approx+10 \mathrm{~d}$, after which the radius either stays constant or decreases slightly. The photospheric expansion velocities based on the blackbody fits up to $+10 \mathrm{~d}$ are $v \approx 1800$ $\mathrm{km} \mathrm{s}^{-1}$ for DES17X1boj and $v \approx 4800 \mathrm{~km} \mathrm{~s}^{-1}$ for DES16E2bjy. Based on the sharp transition in the evolution of radii at roughly +10 


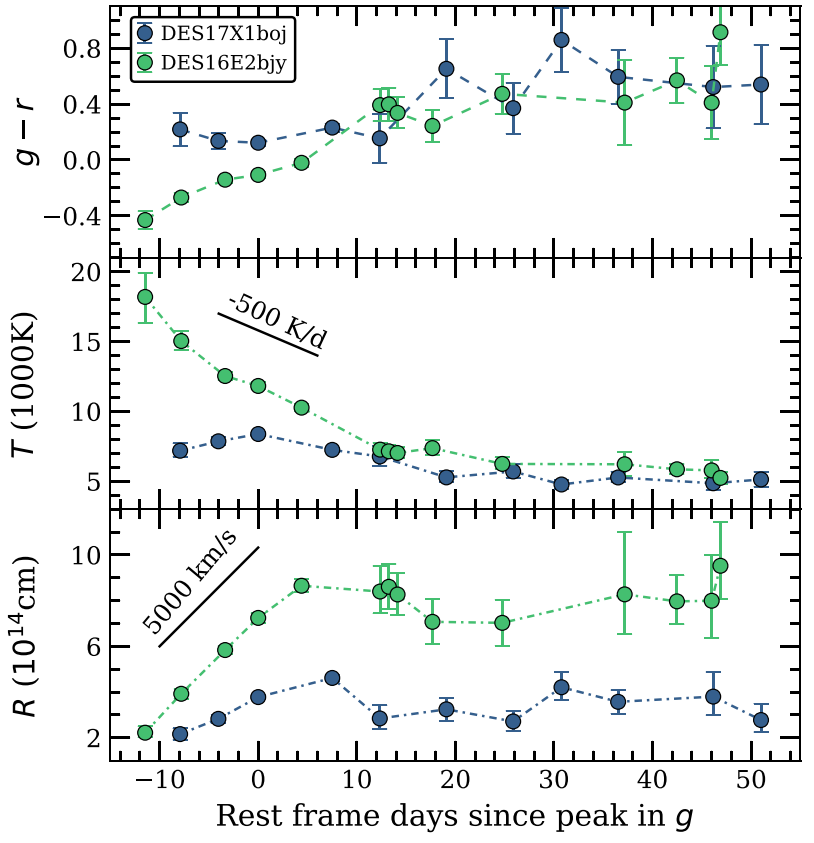

Figure 4. Observed $g-r$ colour (top panel) with temperature (middle panel) and radius evolution (bottom panel) based on blackbody fits to photometry. Errors for temperature and radius are given in $1 \sigma$ confidence. Reference curves for velocity and change of temperature have been added for clarity.

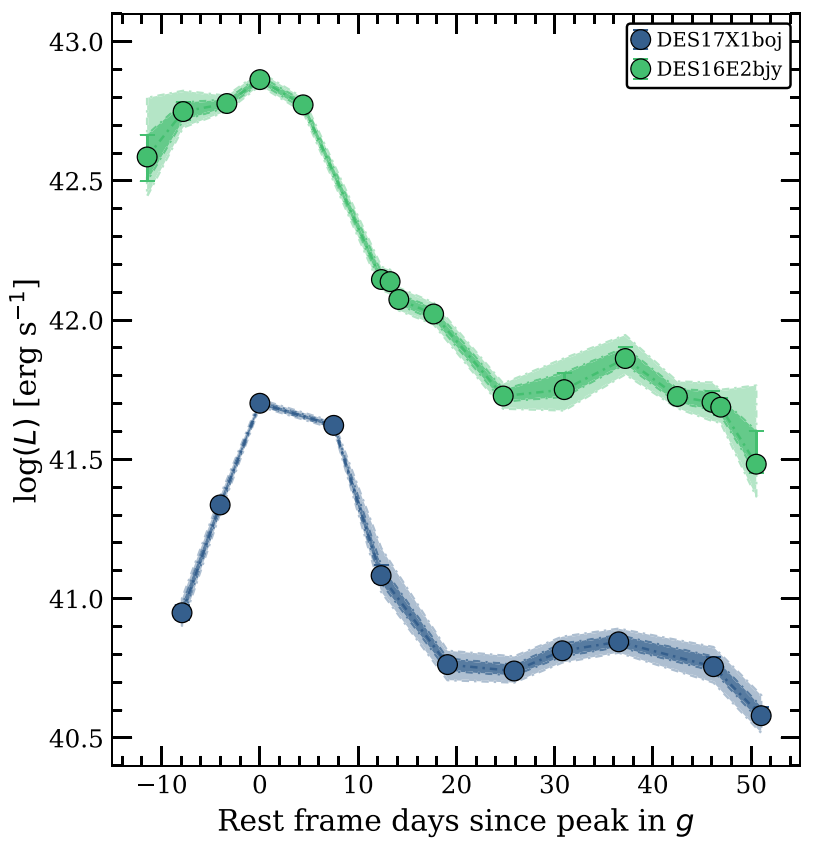

Figure 5. Pseudo-bolometric light curves of DES17X1boj and DES16E2bjy. Light curves are constructed using the blackbody fits and the shaded regions refer to $1 \sigma$ and $2 \sigma$ confidence levels.

$\mathrm{d}$, it is likely that SEDs are not described by blackbody emission alone after this epoch (see Appendix A).

In Fig. 5, we present pseudo-bolometric light curves of DES17X1boj and DES16E2bjy constructed using the blackbody

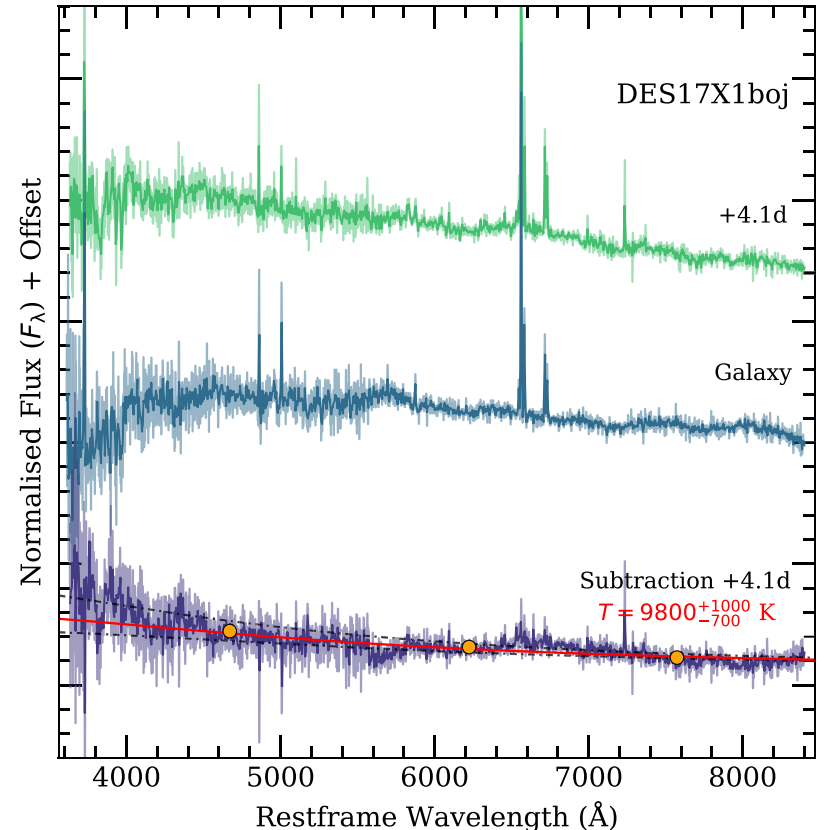

Figure 6. AAT spectrum of DES17X1boj, the host galaxy spectrum, and the resulting host galaxy subtraction (lighter shade) and the corresponding spectra binned by factor of 5 (darker shade). The transient spectrum is taken a few days after the observed peak brightness and holds a considerable amount of transient light. The subtraction shows a blue continuum with blueshifted Ca II absorption feature at $3830 \AA$. The jump at $5600 \AA$ in the subtraction is caused by a dichroic issue in the galaxy spectrum. The red line is the best-fitting blackbody to the subtraction with black dashed lines corresponding to the given $1 \sigma$ errors on the temperature. The orange circles are photometric gri data taken within a few days of the spectrum.

fits (see Appendix A). The double-peaked shape of both light curves is clearly visible in the bolometric light curves. Interestingly, the two-phased rise of the initial peak of DES16E2bjy seen in the griz light curves (see Fig. 2) appears to be slightly more pronounced in the bolometric light curve.

\subsection{Spectroscopic properties}

The AAT spectrum of DES17X1boj is presented in Fig. 6, with the host galaxy spectrum and resulting host galaxy subtraction. The spectrum was obtained $4 \mathrm{~d}$ after the observed maximum brightness, but even at this epoch the host galaxy was 1.0-1.5 mag brighter than the transient in all bands and thus the spectrum was dominated by host galaxy features. After the host galaxy subtraction, the spectrum shows a blue continuum best fit with a blackbody of $T=9800_{-700}^{+1000} \mathrm{~K}$, which is slightly higher than the temperature based on the photometry ( $T \approx 8000 \mathrm{~K}$, see Fig. 4 ). The only significant line feature clearly visible in the subtraction is a relatively broad absorption at $\lambda \approx 3830 \AA$ (measured at the middle of the absorption feature). This feature resembles blueshifted $\mathrm{Ca} H \& \mathrm{~K}$ absorption often seen in various types of SNe [see e.g. SN Ia2011fe (Silverman et al. 2015), SN IIb2011dh (Arcavi et al. 2011), and Ca-rich transient SN2005E (Perets et al. 2010)]. Based on the wavelength at the middle of the feature, the corresponding ejecta velocity would be $v \approx 9400 \mathrm{~km} \mathrm{~s}^{-1}$. The feature has been plotted in Fig. 7, where we also show the best-fitting Gaussian absorption lines. The fitted Gaussian profiles were forced to be at 3813 and $3846 \AA$ (corresponding to ejecta velocity of $v=9400 \mathrm{~km} \mathrm{~s}^{-1}$ ) and 


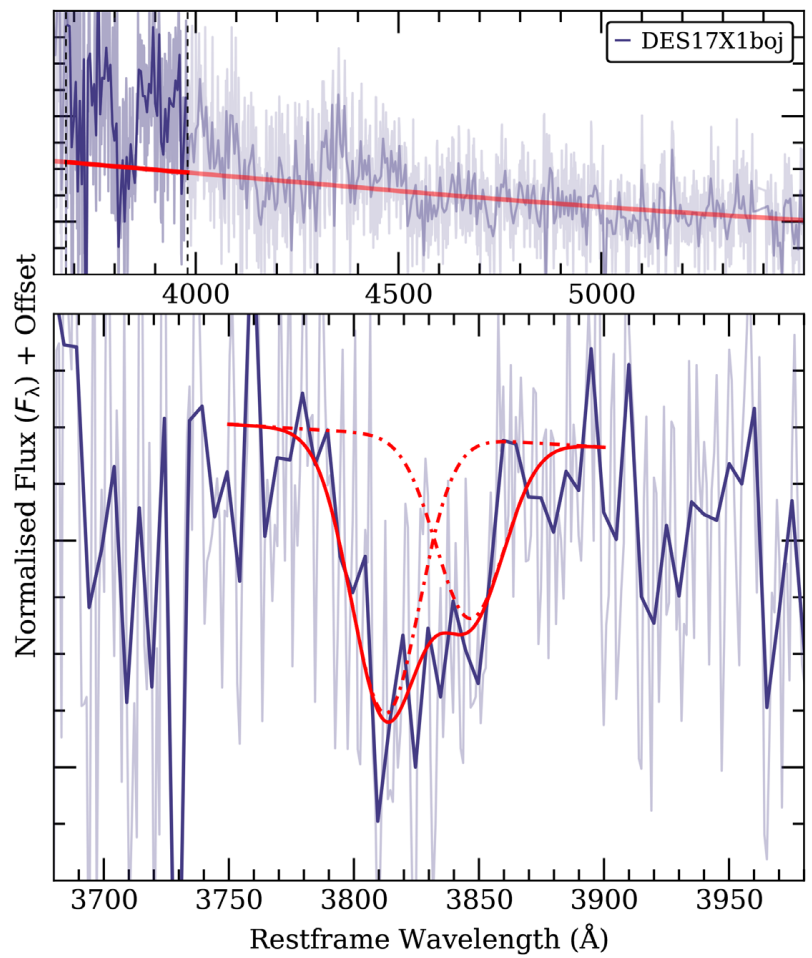

Figure 7. Spectrum of DES17X1boj highlighting the blueshifted $\mathrm{H}$ and $\mathrm{K}$ absorption with the best-fitting blackbody presented in Fig. 6 (top panel) and the absorption feature with best-fitting Gaussian profiles given in red. Lines were fixed to be at 3813 and $3846 \AA$ (corresponding to ejecta velocity of $v \approx$ $9400 \mathrm{~km} \mathrm{~s}^{-1}$ ). The width of the lines was found to be $v_{\text {FWHM }}=2500_{-600}^{+1600}$ $\mathrm{km} \mathrm{s}^{-1}$. Note that the width of the lines was forced to be the same when fitting. The darker shade represents the binned data as per Fig. 6.

to have the same width that was free to vary. The value was found to be $v_{\mathrm{FWHM}}=2500_{-600}^{+1600} \mathrm{~km} \mathrm{~s}^{-1}$, which is significantly higher than the resolution of the instrument $\Delta \lambda \sim 250 \mathrm{~km} \mathrm{~s}^{-1}$ at $\mathrm{H} \alpha$. All given errors were estimated using a Monte Carlo approach with 500 realizations and are shown in $1 \sigma$ confidence.

The high velocity of this blueshifted Ca II absorption is in good agreement with what is seen in SNe and could thus suggest that DES17X1boj is an SN of some type. For instance, in the spectra of SN IIb2011dh, the feature occurs at higher velocity during the rise but it is found at $v \approx 10000 \mathrm{~km} \mathrm{~s}^{-1}$ at the time of peak brightness (Sahu, Anupama \& Chakradhari 2013). On the other hand, the photospheric expansion velocity of this $\mathrm{SN}$ is $v \approx 3000 \mathrm{~km} \mathrm{~s}^{-1}$, estimated based on the evolution of the blackbody radius presented in Ergon et al. (2014). While the value is higher than what was found for DES17X1boj, it demonstrates that a large velocity difference between the photosphere and the absorbing material can occur and has already been observed. Ergon et al. (2014) also estimated photospheric temperature evolution with a peak temperature of $T$ $\approx 8000-9000 \mathrm{~K}$, similar to what is seen in DES17X1boj. Despite the discussed similarities, the light-curve evolution of SN2011dh is smoother and slower than seen in DES17X1boj apart from the short-duration pre-peak often seen in SNe IIb (Arcavi et al. 2011).

In Fig. 6, there seems to be small amount of excess emission above the plotted blackbody fit around $6600 \AA$. To investigate this in detail, we show the spectrum of DES17X1boj around the $\mathrm{H} \alpha$ line in Fig. 8 in comparison with SN II2013ej 19 d after explosion (Dhungana et al. 2016, see also e.g. Valenti et al. 2014; Bose

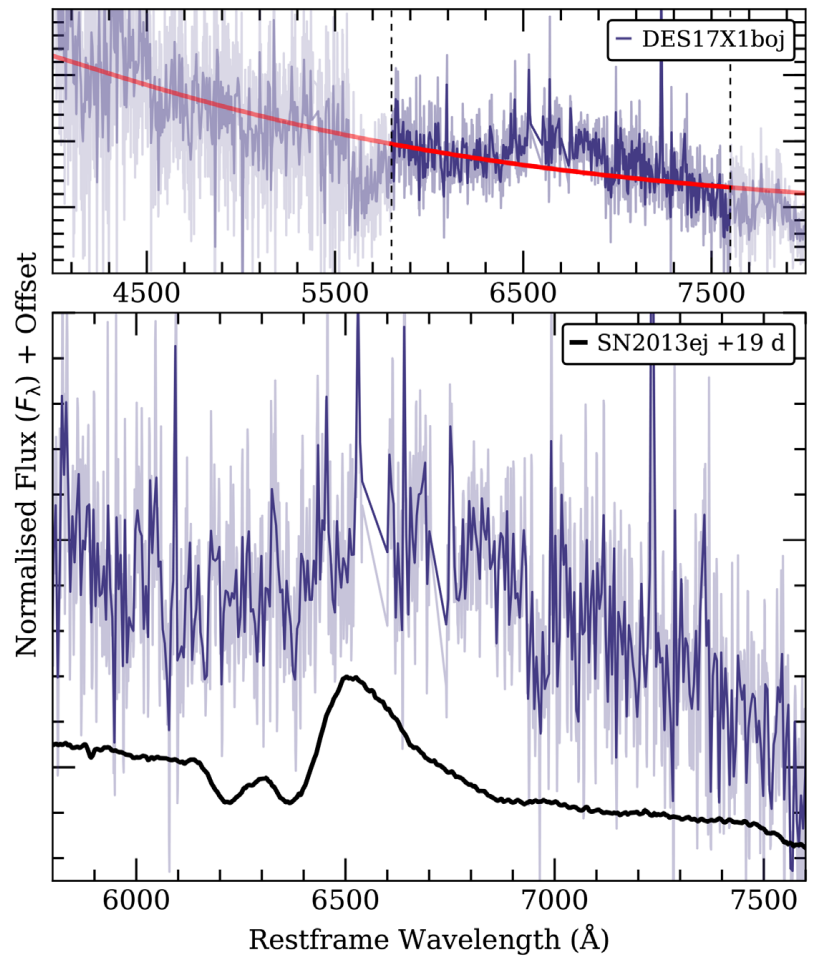

Figure 8. Spectrum of DES17X1boj highlighting the excess emission

around the $\mathrm{H} \alpha$ line on the top and the $\mathrm{H} \alpha$ region of DES17X1boj in comparison with SN II2013ej around peak brightness, $19 \mathrm{~d}$ after the explosion (Dhungana et al. 2016) on the bottom. Solid red line is the bestfitting blackbody presented in Fig. 6. Note that residuals of narrow lines left from the host galaxy subtraction have been masked out. The darker shade represents the binned data as per Fig. 6 .

et al. 2015; Huang et al. 2015). This SN was selected as it has a similar rise time to DES17X1boj in the $r$ band and the spectrum was taken at comparable epoch around the peak brightness. There appears to be excess emission that slightly resembles a broad $\mathrm{H} \alpha$ profile which would indicate that DES17X1boj is an SN II. While the similarity appears to be reasonable, the high level of noise in the spectrum makes it difficult to say anything definite about the feature.

The AAT and SALT spectra of DES16E2bjy, taken shortly after peak brightness, are presented in Fig. 9. Neither of the spectra show other features than underlying blue continuum which is best characterized with a blackbody. A blackbody fit to the AAT spectrum taken at $+0.5 \mathrm{~d}$ after peak brightness in the $g$ band results in a temperature of $T=18400_{-5600}^{+4500} \mathrm{~K}$, which is higher than that based on the blackbody fits to photometry ( $T \approx 12500 \mathrm{~K}$, see Fig. 4 ) due to the high level of noise in the blue part. The SALT spectrum at $+2.7 \mathrm{~d}$ shows a blackbody with $T=11800_{-400}^{+600} \mathrm{~K}$, consistent with the value based on photometry. All presented spectra have been flux calibrated using the DES-SN griz photometry of the transients at the date closest to the epoch of spectra (the maximum difference being 3 d). For DES17X1boj, the aperture photometry of the host from the DES Science Verification (SV; Bonnett et al. 2016; Rykoff et al. 2016) data was also applied. In the case of DES16E2bjy, the host galaxy brightness was estimated to be $m \approx 24.8-26.0$ (depending on the band) in the 2-arcsec aperture. This is significantly fainter than the transient at peak brightness $(m \approx 21)$ when both of the 


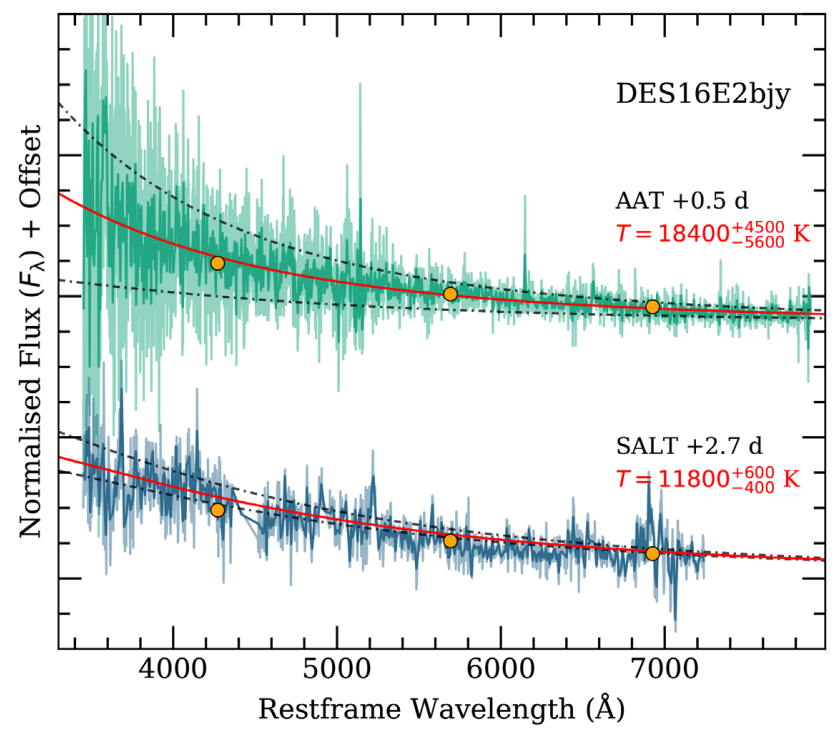

Figure 9. AAT and SALT spectra of DES16E2bjy close to peak brightness. The red line is the best-fitting blackbody to the spectra with the black dashed lines corresponding to the given $1 \sigma$ errors on the temperature. and the orange circles are photometric data points taken within few days of the corresponding spectrum. Note that some strong residuals from sky subtraction have been removed for visual clarity and there is a chip gap in the SALT spectrum around $4400 \AA$.

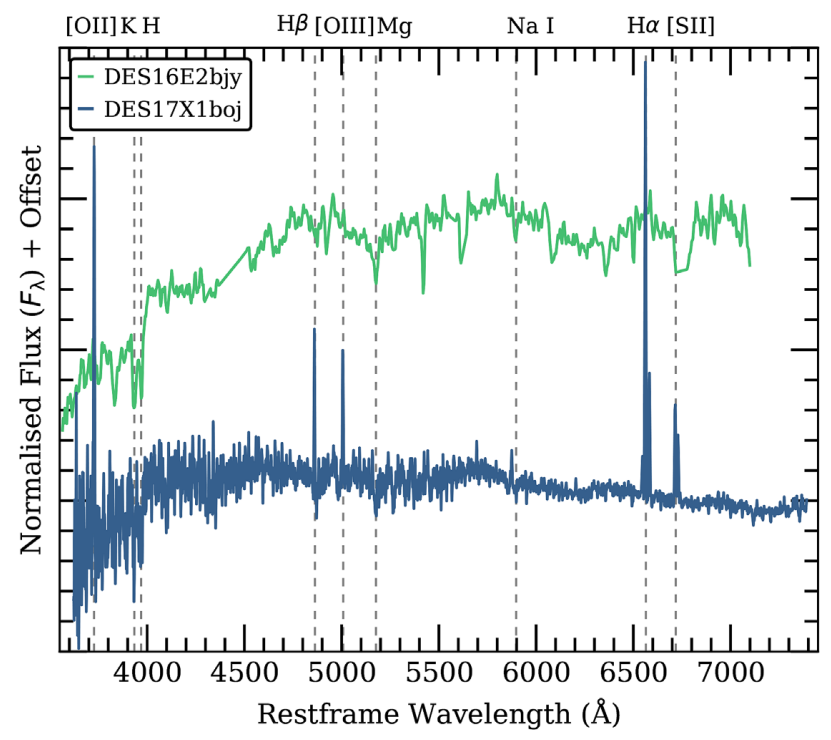

Figure 10. Host spectra of DES17X1boj and DES16E2bjy. Some common absorption and emission lines have been marked with dashed lines.

spectra were taken and thus the contribution of the host galaxy is assumed to be negligible in the spectra.

\section{HOST GALAXY PROPERTIES}

DES17X1boj and DES16E2bjy seem to have occurred in different host environments (see Fig. 1), and to further demonstrate this, we have plotted the host spectra in Fig. 10. The host of DES17X1boj was observed with the AAT under the GAMA programme with a 2-arcsec fibre, positioned at the centre of the host galaxy where the transient also occurred. As DES16E2bjy was separated by 5 arcsec

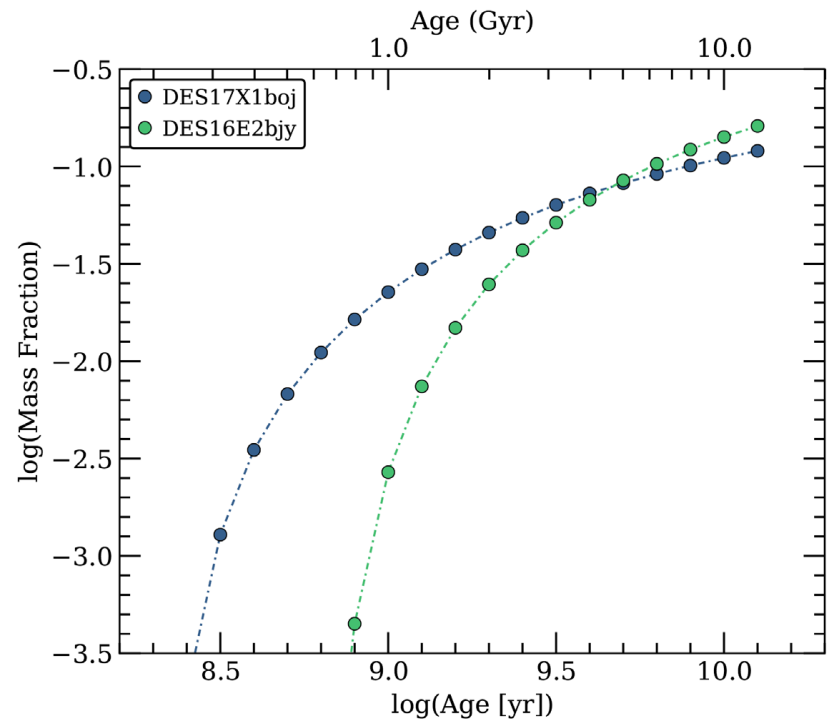

Figure 11. SFH of the best-fitting models for the two transient hosts. The host of DES16E2bjy is clearly older with an average age of the stellar population of $7.6 \mathrm{Gyr}$ and no significant population younger than $1 \mathrm{Gyr}$. The average age in DES17X1boj is 5.4 Gyr.

from its host, we obtained the host spectrum at the core from the long-slit transient spectrum observed with SALT. Based on Fig. 10, it is clear that the hosts of these two transients are different: DES17X1boj happened in a star-forming galaxy characterized by numerous strong nebular emissino lines, while the host of DES16E2bjy is a passive galaxy with no obvious signatures of recent star formation in the optical spectrum (e.g. $\mathrm{H} \alpha$, [O III], $[\mathrm{N}$ II]).

To estimate the star formation history (SFH) of the hosts, we fit the spectra using the PPXF spectral fitting code (Cappellari \& Emsellem 2004; Cappellari 2012, 2017), based on the stellar templates provided by the MILES empirical stellar library (Vazdekis et al. 2010). PPXF simultaneously fits the continuum, stellar absorption features as well as ionised gas emission lines, providing a more robust measure of the emission-line fluxes than a continuum subtraction with a simple flat continuum. The resulting SFHs are presented in Fig. 11. While the host of DES17X1boj consists of younger stellar populations than the host of DES16E2bjy, it still has a dominant population of older, smaller stars, which is the only clear similarity between the two hosts. The presence of old populations can also be clearly seen in the spectrum itself as the spectrum is reasonably red despite the strong, ionized lines that require a young population of stars (see Fig. 10). The age of the young population can be estimated with the equivalent width $W(\mathrm{H} \alpha)=19 \AA$, which indicates the average age of that population to be about $10 \mathrm{Myr}$ or older (see e.g. Kuncarayakti et al. 2016; Xiao et al. 2019). The average age of the continuum stellar population based on the PPXF fit is $5.4 \mathrm{Gyr}$ for the host of DES17X1boj, while for the host of DES16E2bjy, it is $7.6 \mathrm{Gyr}$ with no significant population younger than $1 \mathrm{Gyr}$.

We fit the griz SEDs of the hosts with the CIGALE code (Boquien et al. 2019) to determine the host galaxy masses. We use the Bruzual and Charlot stellar population models (Bruzual \& Charlot 2003) with a Salpeter initial mass function (Salpeter 1955), and model the SFH as delayed with an optional exponential burst. The host mass of DES17X1boj was found to be $\log \left(M / \mathrm{M}_{\odot}\right)=9.76 \pm 0.16$, 
while for DES16E2bjy, it is significantly higher with $\log \left(M / \mathrm{M}_{\odot}\right)=$ $10.46 \pm 0.06$

For the host of DES17X1boj, we also use the flux measurements from the $\mathrm{H}_{\alpha}$, [S II], and [N II] lines to calculate the gas-phase metallicity (Dopita et al. 2016), which results in a slightly sub-solar value of $12+\log (\mathrm{O} / \mathrm{H})=8.51 \pm 0.03$. The gas-phase metallicity for the host of DES16E2bjy can not be estimated due to lack of host galaxy emission lines. We estimated the star formation rate (SFR) for the host of DES17X1boj within the 2-arcsec aperture to be $2.00 \pm 0.03 \cdot 10^{-2} \mathrm{M}_{\odot} \mathrm{yr}^{-1}$ based on the observed $\mathrm{H} \alpha$ flux using the formula of Kennicutt (1998). Extrapolating this over the whole galaxy results in $\mathrm{SFR} \sim 3 \mathrm{M}_{\odot} \mathrm{yr}^{-1}$, corresponding to specific star formation rate $\log (\mathrm{sSFR})=-9.3$. While the given values are likely underestimated due to the central location of the aperture, they demonstrate that the host is moderately star forming.

Apart from the DES-SN optical photometry, the host galaxy of DES16E2bjy has also been detected in the ultraviolet (UV) regime by the Galaxy Evolution Explorer (GALEX; Martin et al. 2005) in both far-UV (FUV, 1350-1750 $\AA$ in observer frame) and near-UV (NUV, and 1750-2750 ̊). The UV magnitudes (FUV = $22.75 \pm 0.07$ and NUV $=22.56 \pm 0.05$ ) correspond to colours FUV $-r=3.99$ and NUV $-r=3.79$, indicating that the host is rather bright in UV for a such passive galaxy in the optical (see e.g. Petty et al. 2013). Assuming that all the UV emission of the galaxy comes from some residual star-formation still occurring in the galaxy, we find SFR $=0.14 \mathrm{M}_{\odot} \mathrm{yr}^{-1}$ (using the formula from Kennicutt 1998) and corresponding specific star formation rate of $\log (\mathrm{sSFR})=-11.3$. While these values demonstrate that some star formation could still be happening in the host, its level is fairly low.

As the location of DES17X1boj is consistent with the nucleus of its host galaxy, it is essential to verify if the host harbours an active galactic nucleus (AGN). In Fig. 12 (top panels), we show that the 5-yr DES-SN light curve of the transient shows no significant variation that would be expected for an AGN. In the same figure, we also show the location of emission-line flux ratios for the host galaxy on the Baldwin-Phillips-Terlevich (BPT) diagram (Baldwin et al. 1981), compared to data from SDSS (Tremonti et al. 2004) and theoretical lines from Kewley et al. (2001) and Kauffmann et al. (2003). BPT diagrams use line ratios (in this case, $[\mathrm{N} \mathrm{II}]_{\lambda 5007} / \mathrm{H} \alpha$ and $[\mathrm{O} \mathrm{III}]_{\lambda 6584} / \mathrm{H} \beta$ ) to distinguish if the line-emitting region has been excited by star formation or an AGN. Based on the diagram, we see that the host of DES17X1boj is found below both of the theoretical lines, indicating that the host does not appear to have an AGN and thus the transient is unlikely to be an AGN flare. In Fig. 12 (bottom panels), we also show that the 5-yr light curve of DES16E2bjy does not exhibit variation either.

Due to the reasonably large separation of the DES16E2bjy and the centre of its assumed host galaxy ( 5 arcsec), it is possible that there is a small satellite galaxy in the vicinity of the passive galaxy that is actually the host. However, while something faint is present at the location of the transient in the DES-SN deep stacks (see Fig. 1), its measured colours match with the passive galaxy and thus it is likely associated with the putative host due to its similar stellar population.

\section{DISCUSSION AND CONCLUSIONS}

In the sections above, we have presented our analysis of the two peculiar DES-SN transients, DES17X1boj and DES16E2bjy, and their host galaxies. Based on the relatively fast photospheric expansion velocities $\left(v \approx 1800 \mathrm{~km} \mathrm{~s}^{-1}\right.$ for DES17X1boj and $v \approx$
$4800 \mathrm{~km} \mathrm{~s}^{-1}$ for DES16E2bjy, based on the blackbody fits) the transients are likely to be either explosive or eruptive in origin. Additionally, the blueshifted Ca II absorption feature identified in the near-peak spectrum of DES17X1boj may imply that it is an $\mathrm{SN}$. As the light curves of the transients evolve very similarly, it is natural to assume that DES17X1boj and DES16E2bjy are products of a similar evolutionary channel. However, the large difference in the peak luminosities and photospheric expansion velocities are difficult to describe under any single scenario. Thus, while in the following we discuss scenarios that could explain the peculiar observational features of both of them, we emphasize that whether these two odd transients originate in similar progenitors is still unclear.

The most striking photometric feature of DES17X1boj and DES16E2bjy is their peculiar double-peaked light-curve evolution. Several CCSNe and SLSNe have been observed to have precursory bumps often credited to a short-lived shock cooling phase early in the light-curve evolution and, in many of these cases, the pre-bumps have actually been as bright or brighter than the 'normal' peak of the SN (see e.g. SN1993J and iPTF14gqr; Wheeler et al. 1993; De et al. 2018). However, these pre-bump features typically last for only $\lesssim 10 \mathrm{~d}$ due to rapidly cooling/expanding material (see e.g. DES14X3taz; Smith et al. 2016b). The main peaks of the two DES$\mathrm{SN}$ events last for roughly $20 \mathrm{~d}$ during which the photospheric radii are clearly increasing. If we assume that these peaks are powered by shock cooling in extended material, we need to explain why they last so long. One possibility could be linked to the photospheric expansion velocities that are not particularly high $\left(v \approx 1800 \mathrm{~km} \mathrm{~s}^{-1}\right.$ for DES17X1boj and $v \approx 4800 \mathrm{~km} \mathrm{~s}^{-1}$ for DES16E2bjy) and thus it is possible that extended material could stay optically thick for such a long period of time to produce the main peaks of the light curves. The secondary peak would then occur after the extended material has dissipated and an underlying slow-rising CCSN would finally emerge. However, while the secondary peak of DES16E2bjy was reasonable for CCSNe at $M_{r} \approx-16, M_{r} \approx-13.5$ of DES17X1boj would be very faint. The faintest known CCSN to date is the SN II1999br at $M=-13.77$ (Pastorello et al. 2004; Anderson et al. 2014). Additionally, having a shock cooling pre-peak that is $2-3$ mag brighter than the main SN would be peculiar. Unfortunately, both of the transients were too faint at the time of the second peak for spectroscopic follow-up and thus we cannot make spectroscopic comparisons at these later epochs.

Our data pose an additional challenge to the shock cooling scenario. While the temperature evolution of DES16E2bjy is rapidly declining from the first detection (as expected for rapidly heated, expanding material, see e.g. iPTF14gqr; De et al. 2018), the temperature of DES17X1boj increases slightly up to the peak brightness. Such an evolution can be explained with shock cooling only if the temperature gradient of the shock heated material is steep enough so that the decreasing opacity could allow us to see deeper layers of the ejecta which are still (despite adiabatic expansion) hotter than the outer layer of the photosphere was at the beginning of the light curve. Whether such a configuration is physically feasible is unclear.

The evolution towards bluer colours and higher temperatures during the rise of a transient is not a particularly rare phenomenon itself and it can be seen in various kinds of transients. SNe Ia become famously bluer in their early light-curve evolution (see, e.g. Burns et al. 2014) as do many stripped envelope SN (SESN, e.g. SN1999ex and SN2010as; Stritzinger et al. 2002; Folatelli et al. 2014, respectively). Furthermore, SN IIb2011dh mentioned earlier shows increasing photospheric temperature for the first $10 \mathrm{~d}$ of the 


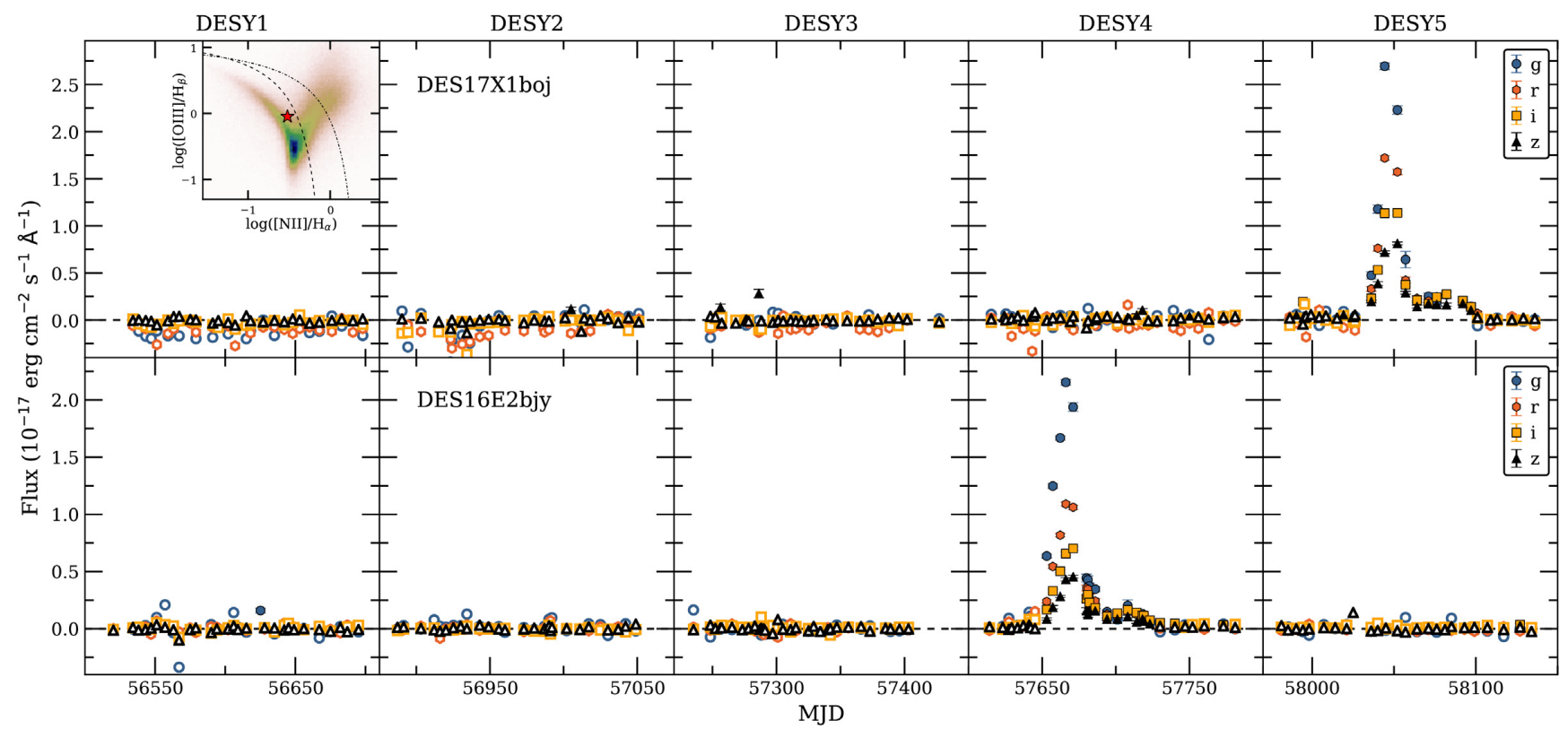

Figure 12. 5-yr flux light curves of DES17X1boj (top panels) and DES16E2bjy (bottom panels). Neither of the transients show significant variation outside the events themselves. A BPT diagram (Baldwin, Phillips \& Terlevich 1981) created with SDSS data (Tremonti et al. 2004) has been plotted for DES17X1boj. The host galaxy, plotted with red star, is located below the theoretical lines from Kewley et al. (2001) and Kauffmann et al. (2003), and thus it is unlikely that the host harbours an AGN. Note that $3 \sigma$ detections are plotted with filled markers and non-detections with open markers and that errors are shown only for the detections.

light curve (Ergon et al. 2014). However, this is not a feature seen only in SNe as, for example, a recent intermediate luminosity red transient (ILRT), M51 OT2019-1, evolved bluer during its rise due to destruction of circumstellar dust in the near vicinity of the very strongly reddened transient (Jencson et al. 2019).

As the spectrum of DES17X1boj shows some broad excess emission around $\mathrm{H} \alpha$ line (see Fig. 8), it is natural to compare the DES transients with SNe II, especially given that there are some photometric similarities as well. For instance, the peak brightnesses fit well within the distribution of SNe II ( $-14 \gtrsim M \gtrsim-19$; see e.g. Anderson et al. 2014; Richardson et al. 2014), the majority of SNe II have plateaus after peak that in same cases show shallow rising (see e.g. SN2009N; Takáts et al. 2014), and both photometry and spectroscopy are well described with a blackbody in the beginning of the light curve. Under this scenario, one would have to assume that the secondary peaks of the DES events would then be plateaus seen in SNe II. However, this is problematic due to the following reasons. The light curves drop 2 mag in 15-20 d in the $r$ band (see Fig. 2) before the start of the secondary peak. Such a decline rate is significantly faster than detected for SNe II (0.9-8.2 mag/100 d in the $V$ band; Anderson et al. 2014; Gutiérrez et al. 2017). The 'plateaus', lasting for $\approx 20 \mathrm{~d}$, would also be on the short side of what would be expected of SNe II (25-72 d in the $V$ band; Anderson et al. 2014). Additionally, the duration of SN II plateaus appear to be correlated with peak brightness so that a shorter plateau is associated with a brighter peak magnitude (Anderson et al. 2014; Galbany et al. 2016). Thus, a short plateau would be unusual for faint a transient such as DES17X1boj. Even if the spectrum of DES17X1boj shows some broad excess around $\mathrm{H} \alpha$, some of the discussed photometric properties of the DES-SN transients do not cohere with observed SNe II. Therefore, if they actually were SNe II, they would have to be peculiar, and given their apparent similarity to each other, they might be first examples of a strange kind of SNe II.
Another interesting type of transient to compare DES17X1boj and DES16E2bjy with are the SN impostors. As shown in Fig. 3, SN2009ip has a short phase of re-brightening around the same phase as the secondary peak of the DES-SN transients, and its peak brightness $\left(M_{\mathrm{V}}=-17.7\right.$; see e.g. Fraser et al. 2013) is similar to DES16E2bjy. However, several other features distinguish our double-peaked DES-SN transients from the SN impostors. While SN2009ip does show re-brightening, its light-curve evolution is clearly different from the DES transients. Additionally, other impostor candidates such as SN2015bh (see e.g. Elias-Rosa et al. 2016) and SN2016bdu (Pastorello et al. 2018) have very similar light curves with SN2009ip, but do not exhibit rebrightening. Furthermore, our photometric data also constrain the long-term variability of DES17X1boj to a level below what was seen in SN2009ip (Pastorello et al. 2013) and SN2016bdu (Pastorello et al. $2018)$ in the years before the brightest event $\left(M_{\mathrm{V}}\right.$ in range -13 to -14). For the more distant event DES16E2bjy, such outbursts would have been below our detection threshold. Regarding the spectroscopic data, the impostors exhibit strong, narrow $\mathrm{H}$ and He lines around peak brightness (see e.g. Fraser et al. 2013; Mauerhan et al. 2013; Pastorello et al. 2013). No such features are seen in either of the DES transients (see Figs 6 and 9), but it is possible that the lines are hidden in the noise.To investigate this, we estimated the limiting equivalent width (EW) for a Gaussianshaped narrow $\mathrm{H} \alpha$ line with $v_{\mathrm{FWHM}}=500 \mathrm{~km} \mathrm{~s}^{-1}$ in our spectra. $\mathrm{H}$ lines with similar widths are often seen in both $\mathrm{SN}$ impostors (Smith et al. 2011) and in SNe IIn (Taddia et al. 2013) where $v_{\text {FWHM }} \sim 100-1000 \mathrm{~km} \mathrm{~s}^{-1}$ are typically measured. For the given configuration, we found limits of EW $\lesssim 5 \AA$ for DES17X1boj and EW $\lesssim 14 \AA$ for DES16E2bjy. In the case of SNe IIn, the line strengths are typically measured in several tens to hundreds of Ångstroms (EW $\gtrsim 40$ Å; Smith, Mauerhan \& Prieto 2014), and thus it is unlikely that narrow $\mathrm{H} \alpha$ lines are hiding in the 
spectra. Due to both photometric and spectroscopic differences, it is unlikely that DES17X1boj and DES16E2bjy are events similar to $\mathrm{SN}$ impostors.

LRNe exhibit long-term light-curve evolution often with several peaks like is the case for SNhunt248 (e.g. Kankare et al. 2015) shown in Fig. 3. LRNe are also photometrically very inhomogeneous class of transients and reach luminosities of $M_{\mathrm{V}} \gtrsim-15$ (e.g. Pastorello et al. 2019) and therefore at least DES17X1boj could be a member of this class. However, the light-curve evolution of LRN is typically significantly slower than what is seen in the DES transients and DES16E2bjy is approximately 3 mag brighter than any classified LRNe. With regards to spectra, many LRNe exhibit strong, narrow lines in their spectra throughout their light curves (see e.g. Pastorello et al. 2019), but not all of them. For instance, for V838 Mon narrow $\mathrm{H} \alpha$ emission was present $5 \mathrm{~d}$ after peak brightness (Smith et al. 2011), but the line was not visible in the decline phase 30-60 d post-peak (Smith et al. 2016a). Thus, while the absence of narrow emission lines in the spectra of the DES transients (see Figs 6 and 9) cannot be used to rule out the LRNe as a possible scenario, different light-curve evolution time-scales and high peak brightness of DES16E2bjy makes it unlikely.

One topic that could give us insight on the origin of the DES-SN transients is where they occurred in their host galaxies. The event location is one of the most constraining observational features about DES16E2bjy: it is found in the outskirts of a passive galaxy. If this galaxy is truly the host of DES16E2bjy, it would disfavour scenarios related to progenitors with massive stars as only few CCSNe have been associated with passive galaxies (see e.g. SNe II Abell399_11_19_0 and SN2016hil and SN Ibn PS112sk; Graham et al. 2012, Irani et al. 2019 and Hosseinzadeh et al. 2019 , respectively). This is almost contradictory to the fact that the photometric and spectroscopic data appears to be well described with a blackbody, which typically requires a significant amount of material that can be shock heated and thus it is a feature often seen in CCSNe. Therefore, we conclude one of the following must be true: either there has been a small amount of recent star formation in the host galaxy leading to this peculiar transient, or this is a new type of transient that originates in an environment with old stellar populations. As discussed in Section 4, the $U V-r$ colours are higher than expected from from early-type galaxies, potentially suggesting that residual star formation is still occurring in the galaxy (see e.g. Petty et al. 2013). Therefore, it is not possible to conclusively determine if DES16E2bjy is a product of an old stellar population. DES17X1boj, on the other hand, is associated with a star-forming galaxy with a non-negligible old stellar population, so the transient could originate from either young or old stellar populations.

As DES16E2bjy appears to be associated with a passive galaxy, one also has to consider a thermonuclear origin for it. However, as seen in Fig. 3, its light-curve shape is completely different when compared with prototypical SN Ia2011fe, even if its absolute magnitude is loosely within the Ia distribution $(-18 \gtrsim M \gtrsim-201$ see e.g. Richardson et al. 2014). Comparison with SNe Iax, the largest class of peculiar thermonuclear SNe also known as SN2002cx-like (Li et al. 2003; Jha et al. 2006; Foley et al. 2013), yields a similar result. While their peak magnitudes are lower than for 'normal' SNe Ia ( $-13 \gtrsim M \gtrsim-19$; see e.g. Jha 2017), they have no stronger secondary peaks powered by Fe III recombination seen in SNe Ia and therefore they are clearly different than DES16E2bjy. Additionally, the colour of SNe Iax is significantly redder than for DES16E2bjy in the beginning of the light curve $(B-V \gtrsim 0.0$; Foley et al. 2013). Due to the different light-curve shapes and inconsistent colours, it is unlikely that thermonuclear $\mathrm{SN}$ alone can be responsible for DES16E2bjy and even less for DES17X1boj due to its lower peak magnitude.

It is possible that the DES-SN transients are thermonuclear with addition of some other power source, for instance interaction with circumstellar material (CSM). Under this scenario, the thermonuclear explosion cannot be a standard SN Ia as they are too bright, so it would have to be a peculiar one such as SNe Iax. However, as these appear to be too red in the beginning of the light curve, the interaction would have to be such that it causes the beginning of the light curve to be bluer and still produces the secondary peak. While no narrow emission lines are present in the peak spectra of the DES transients, the $\mathrm{EW}$ of narrow $\mathrm{H} \alpha$ emission in the interacting $\mathrm{SNe}$ Ia (Ia-CSM) is not statistically different than for SNe IIn (Silverman et al. 2013). Thus, based on the limits of the emission we estimated, it is unlikely that there is a significant amount of interaction at the time of peak brightness. The presence of interaction cannot be ruled out during the secondary peaks, as there was no spectral coverage during these epochs. However, due to the needed strong effect of interaction on the light curves of the DES transients, this particular scenario of thermonuclear SNe with CSM interaction sounds contrived. Therefore, we consider it unlikely but it is possible that some other power source in addition to peculiar thermonuclear $\mathrm{SNe}$ could produce these transients.

DES17X1boj occurred in a star-forming galaxy so it is possible that at least some of the brightness difference between the two transients could be explained by host galaxy extinction. However, the blue bands (especially $g$ ) are bright and persistent throughout the light curves (see Fig. 2) and the the blackbody fits are very good in the beginning of the light curve (see Fig. A1), neither of which would be expected in case of strong extinction. Thus, while it is likely that there is some extinction in the host galaxy, meaning DES17X1boj is intrinsically bluer and brighter, it does not seem probable that the difference in brightness between the two transients is caused by that.

As both DES-SN events were found at relatively low redshifts and as DES16E2bjy is reasonably bright at peak, it is interesting that no transients with similar light-curve evolution have been identified in the literature, especially if we want to assume that they are powered by same physical mechanism. However, as the main characterizing feature is the faint, secondary peak seen in all four griz bands some of these transients may have gone by unrecognized. This argument can be put in context with the example of Ca-rich transients described by absolute magnitudes of -15 to -16.5 . Even though the first such event was published nearly a decade ago (SN2005E; Perets et al. 2010), their total number has stayed low $(\sim 10)$ even if their volumetric rate in local universe has been estimated to be significant fraction of the Ia rate ( $\gtrsim 10$ per cent; Perets et al. 2010; Frohmaier et al. 2018). This demonstrates that even if transients such as DES17X1boj or DES16E2bjy were relatively common, the faintness of the secondary peak makes them difficult to separate from the general SN population.

Here we have presented our analysis of the two peculiar doublepeaked DES-SN transients, DES17X1boj and DES16E2bjy. As discussed earlier in this section, any standard SN scenario creates more conflicts than it solves. While early photometric and spectroscopic data would lean towards an origin related to CCSNe, it is difficult to explain why such a peculiar CCSN would be found in a passive galaxy. While this conflict might convince one that these transients must then be thermonuclear, their secondary peaks cannot be produced via means of Fe III recombination like is the case for thermonuclear $\mathrm{SNe}$, making this scenario contrived as well. It is also 
entirely possible that, despite nearly identical light curves and the fact that no similar transients are found in the literature, the events are not physically similar to each other and are, in fact, produced via different progenitor channels. More similar transients need to be discovered to verify if they are truly similar, and if so, what the progenitors are like.

\section{ACKNOWLEDGEMENTS}

We thank the anonymous referee for the helpful feedback and Andrea Pastorello for fruitful discussion. We acknowledge support from the European Union's 7th Framework Programme (EU/FP7) European Research council (ERC) grant 615929, and Science and Technology Facilities Council (STFC) grant ST/R000506/1. LG was funded by the European Union's Horizon 2020 research and innovation programme under the Marie Skłodowska-Curie grant agreement No. 839090.

Some of the observations reported in this paper were obtained with the Southern African Large Telescope (SALT) and the Galaxy and Mass Assembly (GAMA) programme. GAMA is a joint European-Australasian project based around a spectroscopic campaign using the Anglo-Australian Telescope. The GAMA input catalogue is based on data taken from the Sloan Digital Sky Survey and the UKIRT Infrared Deep Sky Survey. Complementary imaging of the GAMA regions is being obtained by a number of independent survey programmes including GALEX MIS, VST KiDS, VISTA VIKING, WISE, Herschel-ATLAS, GMRT, and ASKAP, providing UV to radio coverage. GAMA is funded by the STFC (UK), the ARC (Australia), the AAO, and the participating institutions. The GAMA website is http://www.gama-survey.org/.

Funding for the DES Projects has been provided by the US Department of Energy, the US National Science Foundation, the Ministry of Science and Education of Spain, the Science and Technology Facilities Council of the United Kingdom, the Higher Education Funding Council for England, the National Center for Supercomputing Applications at the University of Illinois at Urbana-Champaign, the Kavli Institute of Cosmological Physics at the University of Chicago, the Center for Cosmology and Astro-Particle Physics at the Ohio State University, the Mitchell Institute for Fundamental Physics and Astronomy at Texas A\&M University, Financiadora de Estudos e Projetos, Fundação Carlos Chagas Filho de Amparo à Pesquisa do Estado do Rio de Janeiro, Conselho Nacional de Desenvolvimento Científico e Tecnológico and the Ministério da Ciência Tecnologia e Inovação, the Deutsche Forschungsgemeinschaft, and the Collaborating Institutions in the Dark Energy Survey.

The Collaborating Institutions are Argonne National Laboratory, the University of California at Santa Cruz, the University of Cambridge, Centro de Investigaciones Energéticas, Medioambientales y Tecnológicas-Madrid, the University of Chicago, University College London, the DES-Brazil Consortium, the University of Edinburgh, the Eidgenössische Technische Hochschule (ETH) Zürich, Fermi National Accelerator Laboratory, the University of Illinois at Urbana-Champaign, the Institut de Ciències de l'Espai (IEEC/CSIC), the Institut de Física d'Altes Energies, Lawrence Berkeley National Laboratory, the Ludwig-Maximilians Universität München and the associated Excellence Cluster Universe, the University of Michigan, the National Optical Astronomy Observatory, the University of Nottingham, the Ohio State University, the University of Pennsylvania, the University of Portsmouth, SLAC National Accelerator Laboratory, Stanford University, the
University of Sussex, Texas A\&M University, and the OzDES Membership Consortium.

This paper is based in part on observations at Cerro Tololo InterAmerican Observatory, National Optical Astronomy Observatory, which is operated by the Association of Universities for Research in Astronomy (AURA) under a cooperative agreement with the National Science Foundation, and in part on data acquired at the Anglo-Australian Telescope, under program A/2013B/012. We acknowledge the traditional owners of the land on which the AAT stands, the Gamilaraay people, and pay our respects to elders past and present.

The DES data management system is supported by the National Science Foundation under Grant Numbers AST-1138766 and AST-1536171. The DES participants from Spanish institutions are partially supported by MINECO under grants AYA201571825, ESP2015-66861, FPA2015-68048, SEV-2016-0588, SEV2016-0597, and MDM-2015-0509, some of which include ERDF funds from the European Union. IFAE is partially funded by the CERCA programme of the Generalitat de Catalunya. Research leading to these results has received funding from the European Research Council under the European Union's Seventh Framework Programme (FP7/2007-2013) including ERC grant agreements 240672, 291329, and 306478. We acknowledge support from the Brazilian Instituto Nacional de Ciência e Tecnologia (INCT) eUniverse (CNPq grant 465376/2014-2).

This paper has been authored by Fermi Research Alliance, LLC under Contract No. DE-AC02-07CH11359 with the US Department of Energy, Office of Science, Office of High Energy Physics. The United States Government retains and the publisher, by accepting the article for publication, acknowledges that the United States Government retains a non-exclusive, paid-up, irrevocable, worldwide license to publish or reproduce the published form of this manuscript, or allow others to do so, for United States Government purposes.

This research used resources of the National Energy Research Scientific Computing Center (NERSC), a US Department of Energy Office of Science User Facility operated under Contract No. DEAC02-05CH11231.

\section{REFERENCES}

Ambikasaran S., Foreman-Mackey D., Greengard L., Hogg D. W., O’Neil M., 2016, IEEE Trans. Pattern Anal. Mach. Intell., 38, 252

Anderson J. P. et al., 2014, ApJ, 786, 67

Angus C. R. et al., 2019, MNRAS, 487, 2215

Arcavi I. et al., 2011, ApJ, 742, L18

Baldry I. K. et al., 2018, MNRAS, 474, 3875

Baldwin J. A., Phillips M. M., Terlevich R., 1981, PASP, 93, 5

Bonnett C. et al., 2016, Phys. Rev. D, 94, 042005

Boquien M., Burgarella D., Roehlly Y., Buat V., Ciesla L., Corre D., Inoue A. K., Salas H., 2019, A\&A, 622, A103

Bose S. et al., 2015, ApJ, 806, 160

Brouty M., Dubois P., Theureau G., Paturel G., Rousseau J., Petit C., Prugniel P., Cambrésy L., 2003, A\&A, 412, 45

Brown P. J., Breeveld A. A., Holland S., Kuin P., Pritchard T., 2014, Ap\&SS, 354,89

Bruzual G., Charlot S., 2003, MNRAS, 344, 1000

Burns C. R. et al., 2014, ApJ, 789, 32

Cappellari M., 2012, Astrophysics Source Code Library, record ascl: 1210.002

Cappellari M., 2017, MNRAS, 466, 798

Cappellari M., Emsellem E., 2004, PASP, 116, 138

Childress M. J. et al., 2017, MNRAS, 472, 273

Crawford S. M. et al., 2010, SPIE Astron. Instrum., 7737, 773725 
D'Andrea C. B. et al., 2018, preprint (arXiv:1811.09565)

De K. et al., 2018, Science, 362, 201

Dhungana G. et al., 2016, ApJ, 822, 6

Dopita M. A., Kewley L. J., Sutherland R. S., Nicholls D. C., 2016, Ap\&SS, 361,61

Driver S. P. et al., 2009, Astron. Geophys., 50, 12

Drout M. R. et al., 2014, ApJ, 794, 23

Elias-Rosa N. et al., 2016, MNRAS, 463, 3894

Ergon M. et al., 2014, A\&A, 562, A17

Filippenko A. V., 1997, ARA\&A, 35, 309

Flaugher B. et al., 2015, AJ, 150, 150

Folatelli G. et al., 2014, ApJ, 792, 7

Foley R. J. et al., 2013, ApJ, 767, 57

Fraser M. et al., 2013, MNRAS, 433, 1312

Frohmaier C., Sullivan M., Maguire K., Nugent P., 2018, ApJ, 858, 50

Gal-Yam A., 2017, Handbook of Supernovae. Springer, Cham, p. 195

Galbany L. et al., 2016, AJ, 151, 33

Graham M. L. et al., 2012, ApJ, 753, 68

Guillochon J., Parrent J., Kelley L. Z., Margutti R., 2016, ApJ, 835, 64

Gutiérrez C. P. et al., 2017, ApJ, 850, 90

Hosseinzadeh G. et al., 2018, ApJ, 861, 63

Hosseinzadeh G., McCully C., Zabludoff A. I., Arcavi I., French K. D., Howell D. A., Berger E., Hiramatsu D., 2019, ApJ, 871, L9

Howell D. A., 2017, Handbook of Supernovae. Springer, Cham, p. 431

Hsiao E. Y., Conley A., Howell D. A., Sullivan M., Pritchet C. J., Carlberg R. G., Nugent P. E., Phillips M. M., 2007, ApJ, 663, 1187

Huang F. et al., 2015, ApJ, 807, 59

Inserra C. et al., 2018, MNRAS, 475, 1046

Irani I. et al., 2019, ApJ, 887, 10

Jencson J. E. et al., 2019, ApJ, 880, L20

Jha S. W., 2017, Handbook of Supernovae. Springer, Cham, p. 375

Jha S., Branch D., Chornock R., Foley R. J., Li W., Swift B. J., Casebeer D., Filippenko A. V., 2006, AJ, 132, 189

Kankare E. et al., 2015, A\&A, 581, L4

Kasen D., 2006, ApJ, 649, 939

Kauffmann G. et al., 2003, MNRAS, 346, 1055

Kennicutt R. C., 1998, ApJ, 498, 541

Kessler R. et al., 2015, AJ, 150, 172

Kewley L. J., Dopita M. A., Sutherland R. S., Heisler C. A., Trevena J., 2001, ApJ, 556, 121

Kuncarayakti H., Galbany L., Anderson J. P., Krühler T., Hamuy M., 2016, A\&A, 593, A78

Leloudas G. et al., 2012, A\&A, 541, A129

Li W. et al., 2003, PASP, 115, 453

Martin D. C. et al., 2005, ApJ, 619, L1

Mauerhan J. C. et al., 2013, MNRAS, 430, 1801

Mauerhan J. C. et al., 2015, MNRAS, 447, 1922

Mauerhan J. C., Van Dyk S. D., Johansson J., Fox O. D., Filippenko A. V., Graham M. L., 2018, MNRAS, 473, 3765

Munari U., Henden A., Belligoli R., Castellani F., Cherini G., Righetti G. L., Vagnozzi A., 2013, New Astron., 20, 30
Nakaoka T. et al., 2018, ApJ, 859, 78

Nordsieck K. H., Burgh E. B., Kobulnicky H. A., Williams T. B., O’Donoghue D., Percival J. W., Smith M. P., 2001, Bull. Am. Astron. Soc., 33, 1465

Nugent P., Kim A., Perlmutter S., 2002, PASP, 114, 803

Pastorello A. et al., 2004, MNRAS, 347, 74

Pastorello A. et al., 2013, ApJ, 767, 1

Pastorello A. et al., 2018, MNRAS, 474, 197

Pastorello A., Mason E., Taubenberger S., Fraser M., Cortini G., Tomasella L., Botticella M. T., 2019, A\&A, 630, A75

Perets H. B. et al., 2010, Nature, 465, 322

Petty S. M. et al., 2013, AJ, 146, 77

Pursiainen M. et al., 2018, MNRAS, 481, 894

Quimby R. M. et al., 2011, Nature, 474, 487

Richardson D., Jenkins R. L. III, Wright J., Maddox L., 2014, AJ, 147, 118

Richmond M. W., Smith H. A., 2012, J. Am. Assoc. Var. Star Obs., 40, 872

Rykoff E. S. et al., 2016, ApJS, 224, 1

Sahu D. K., Anupama G. C., Chakradhari N. K., 2013, MNRAS, 433, 2

Salpeter E. E., 1955, ApJ, 121, 161

Schlafly E. F., Finkbeiner D. P., 2011, ApJ, 737, 103

Silverman J. M. et al., 2013, ApJS, 207, 3

Silverman J. M., Vinkó J., Marion G. H., Craig Wheeler J., Barna B., Szalai T., Mulligan B. W., Filippenko A. V., 2015, MNRAS, 451, 1973

Smith N., Li W., Silverman J. M., Ganeshalingam M., Filippenko A. V., 2011, MNRAS, 415, 773

Smith N., Mauerhan J. C., Prieto J. L., 2014, MNRAS, 438, 1191

Smith N. et al., 2016a, MNRAS, 458, 950

Smith M. et al., 2016b, ApJ, 818, L8

Smith M. et al., 2018, ApJ, 854, 37

Stritzinger M. et al., 2002, AJ, 124, 2100

Taddia F. et al., 2013, A\&A, 555, A10

Takáts K. et al., 2014, MNRAS, 438, 368

Tremonti C. A. et al., 2004, ApJ, 613, 898

Tsvetkov D. Y., Shugarov S. Y., Volkov I. M., Goranskij V. P., Pavlyuk N. N., Katysheva N. A., Barsukova E. A., Valeev A. F., 2013, Contrib. Astron. Obs. Skaln. Pleso, 43, 94

Valenti S. et al., 2014, MNRAS, 438, L101

Vazdekis A., Sánchez-Blázquez P., Falcón-Barroso J., Cenarro A. J., Beasley M. A., Cardiel N., Gorgas J., Peletier R. F., 2010, MNRAS, 404, 1639

Wheeler J. C. et al., 1993, ApJ, 417, L71

Wiseman P. et al., 2020, preprint (arXiv:2001.02640)

Xiao L., Galbany L., Eldridge J. J., Stanway E. R., 2019, MNRAS, 482, 384

Yuan F. et al., 2015, MNRAS, 452, 3047

\section{APPENDIX A: BLACKBODY FITS}

The blackbody fits to the data of DES17X1boj and DES16E2bjy are shown in Figs A1 and A2, respectively. 


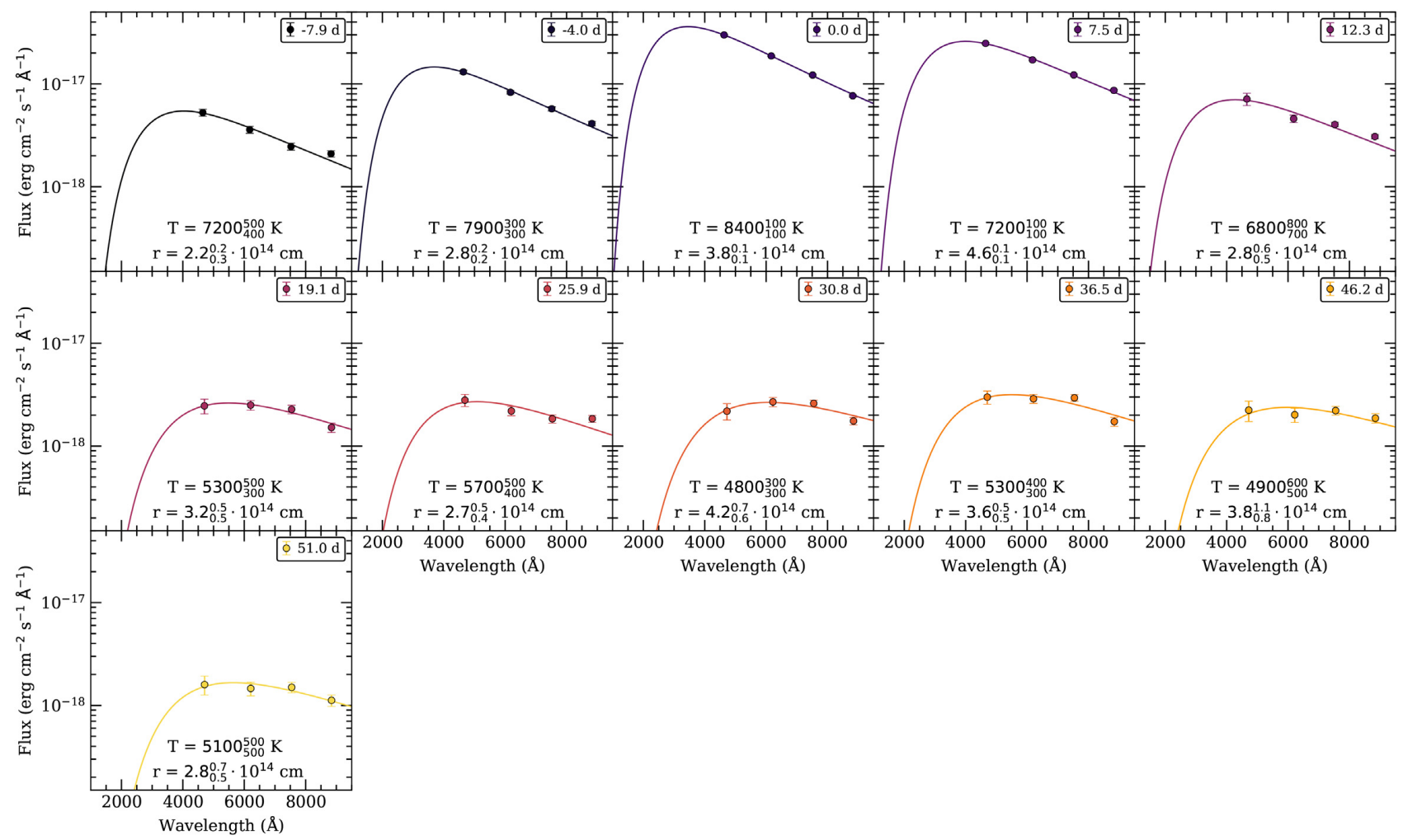

Figure A1. Blackbody fits to photometry of DES17X1boj for every epoch with griz detections. Best-fitting temperatures and radii are given with $1 \sigma$ uncertainties.

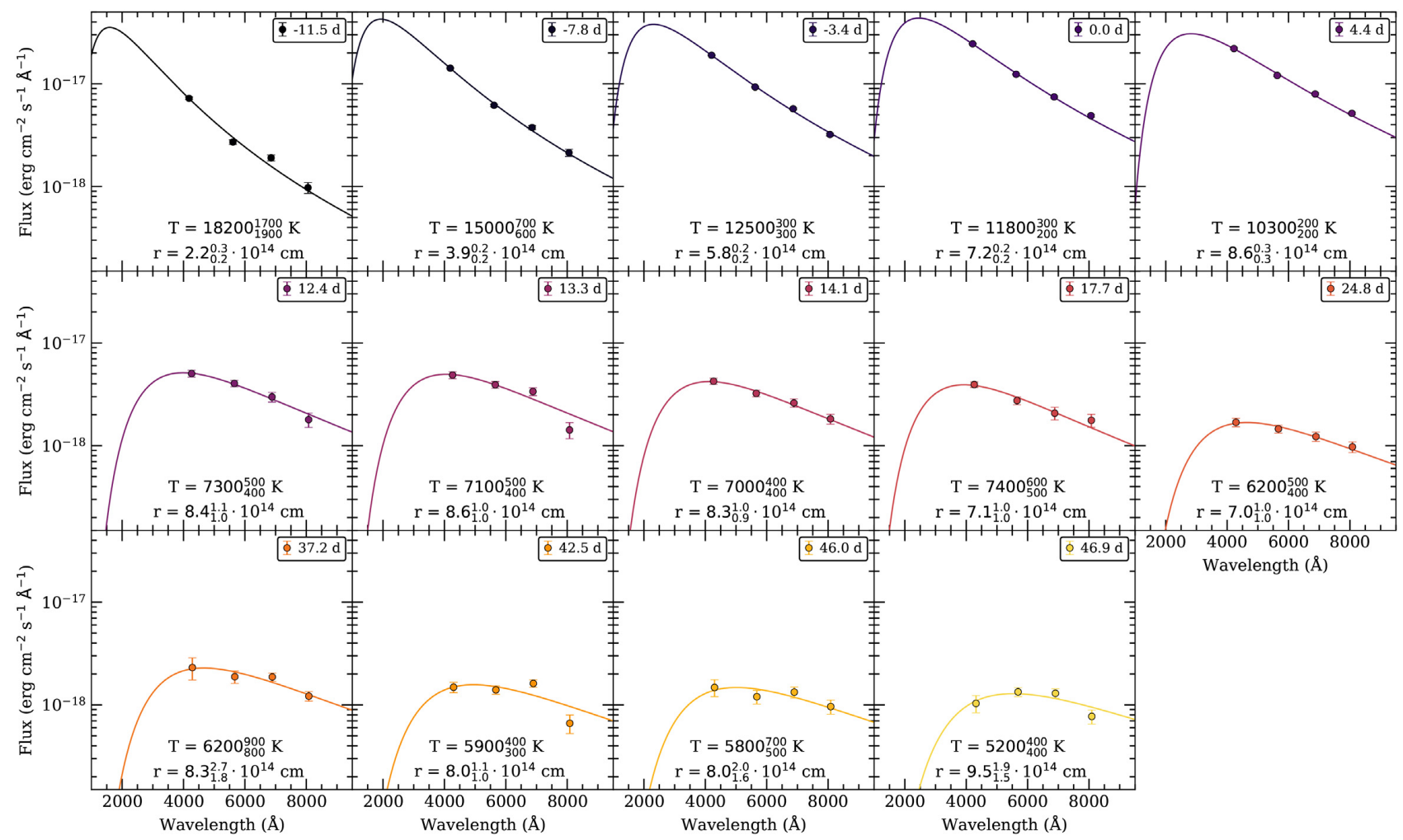

Figure A2. Blackbody fits to photometry of DES16E2bjy for every epoch with griz detections. Best-fitting temperatures and radii are given with $1 \sigma$ uncertainties. 
${ }^{1}$ School of Physics and Astronomy, University of Southampton, Southampton SO17 1BJ, UK

${ }^{2}$ Institute of Cosmology and Gravitation, University of Portsmouth, Portsmouth PO1 3FX, UK

${ }^{3}$ Departamento de Física Teórica y del Cosmos, Universidad de Granada, E-18071 Granada, Spain

${ }^{4}$ Lawrence Berkeley National Laboratory, 1 Cyclotron Road, Berkeley, CA 94720, USA

${ }^{5}$ African Institute for Mathematical Sciences, 6 Melrose Road, Muizenberg 7945, South Africa

${ }^{6}$ South African Astronomical Observatory, PO Box 9, Observatory 7935, South Africa

${ }^{7}$ Department of Physics and Astronomy, University of Pennsylvania, Philadelphia, PA 19104, USA

${ }^{8}$ INAF, Astrophysical Observatory of Turin, I-10025 Pino Torinese, Italy

${ }^{9}$ School of Mathematics and Physics, University of Queensland, Brisbane, QLD 4072, Australia

${ }^{10}$ Santa Cruz Institute for Particle Physics, Santa Cruz, CA 95064, USA

${ }^{11}$ School of Physics and Astronomy, Cardiff University, Queens Buildings, The Parade, Cardiff CF24 3AA, UK

${ }^{12}$ Department of Astronomy and Astrophysics, University of Chicago, Chicago, IL 60637, USA

${ }^{13}$ Kavli Institute for Cosmological Physics, University of Chicago, Chicago, IL 60637, USA

${ }^{14}$ Sydney Institute for Astronomy, School of Physics, A28, The University of Sydney, NSW 2006, Australia

${ }^{15}$ The Research School of Astronomy and Astrophysics, Australian National University, ACT 2601, Australia

${ }^{16}$ Université Clermont Auvergne, CNRS/IN2P3, LPC, F-63000 ClermontFerrand, France

${ }^{17}$ Department of Physics, Duke University, Durham, NC 27708, USA

${ }^{18}$ Department of Astronomy, University of Texas at Austin, Austin, TX 78712 , USA

${ }^{19}$ Cerro Tololo Inter-American Observatory, National Optical Astronomy Observatory, Casilla 603, La Serena, Chile

${ }^{20}$ Fermi National Accelerator Laboratory, PO Box 500, Batavia, IL 60510, USA

${ }^{21}$ Instituto de Fisica Teorica UAM/CSIC, Universidad Autonoma de Madrid, E-28049 Madrid, Spain

${ }^{22}$ CNRS, UMR 7095, Institut d'Astrophysique de Paris, F-75014 Paris, France

${ }^{23}$ Sorbonne Universités, UPMC Université Paris 06, UMR 7095, Institut d'Astrophysique de Paris, F-75014 Paris, France

${ }^{24}$ Department of Physics and Astronomy, University College London, Gower Street, London WC1E 6BT, UK

${ }^{25}$ Kavli Institute for Particle Astrophysics and Cosmology, PO Box 2450, Stanford University, Stanford, CA 94305, USA

${ }^{26}$ SLAC National Accelerator Laboratory, Menlo Park, CA 94025, USA
${ }^{27}$ Centro de Investigaciones Energéticas, Medioambientales y Tecnológicas (CIEMAT), E-28040 Madrid, Spain

${ }^{28}$ Laboratório Interinstitucional de e-Astronomia - LIneA, Rua Gal. José Cristino 77, Rio de Janeiro, RJ-20921-400, Brazil

${ }^{29}$ Department of Astronomy, University of Illinois at Urbana-Champaign, 1002 W. Green Street, Urbana, IL 61801, USA

${ }^{30}$ National Center for Supercomputing Applications, 1205 West Clark St, Urbana, IL 61801, USA

${ }^{31}$ Observatório Nacional, Rua Gal. José Cristino 77, Rio de Janeiro, RJ20921-400, Brazil

${ }^{32}$ Department of Physics, IIT Hyderabad, Kandi, Telangana 502285, India

${ }^{33}$ Department of Astronomy/Steward Observatory, University of Arizona, 933 North Cherry Avenue, Tucson, AZ 85721-0065, USA

${ }^{34}$ Jet Propulsion Laboratory, California Institute of Technology, 4800 Oak Grove Dr., Pasadena, CA 91109, USA

${ }^{35}$ Institut d'Estudis Espacials de Catalunya (IEEC), E-08034 Barcelona, Spain

${ }^{36}$ Institute of Space Sciences (ICE, CSIC), Campus UAB, Carrer de Can Magrans, s/n, E-08193 Barcelona, Spain

${ }^{37}$ Department of Astronomy, University of Michigan, Ann Arbor, MI 48109, USA

${ }^{38}$ Department of Physics, University of Michigan, Ann Arbor, MI 48109, USA

${ }^{39}$ Department of Physics, Stanford University, 382 Via Pueblo Mall, Stanford, CA 94305, USA

${ }^{40}$ Center for Cosmology and Astro-Particle Physics, The Ohio State University, Columbus, $\mathrm{OH} 43210$, USA

${ }^{41}$ Department of Physics, The Ohio State University, Columbus, OH 43210 , USA

${ }^{42}$ Center for Astrophysics $\mid$ Harvard and Smithsonian, 60 Garden Street, Cambridge, MA 02138, USA

${ }^{43}$ Australian Astronomical Optics, Macquarie University, North Ryde, NSW 2113, Australia

${ }^{44}$ Lowell Observatory, 1400 Mars Hill Rd, Flagstaff, AZ 86001, USA

${ }^{45}$ George P. and Cynthia Woods Mitchell Institute for Fundamental Physics and Astronomy, and Department of Physics and Astronomy, Texas A\&M University, College Station, TX 77843, USA

${ }^{46}$ Institució Catalana de Recerca i Estudis Avançats, E-08010 Barcelona, Spain

${ }^{47}$ Institut de Física d'Altes Energies (IFAE), The Barcelona Institute of Science and Technology, Campus UAB, E-08193 Bellaterra (Barcelona), Spain

${ }^{48}$ Department of Astrophysical Sciences, Princeton University, Peyton Hall, Princeton, NJ 08544, USA

${ }^{49}$ Computer Science and Mathematics Division, Oak Ridge National Laboratory, Oak Ridge, TN 37831, USA

This paper has been typeset from a $\mathrm{T}_{\mathrm{E}} \mathrm{X} / \mathrm{L} \mathrm{T} \mathrm{E} \mathrm{X}$ file prepared by the author. 